American Journal of

Health, Medicine and Nursing Practice (AJHMN)

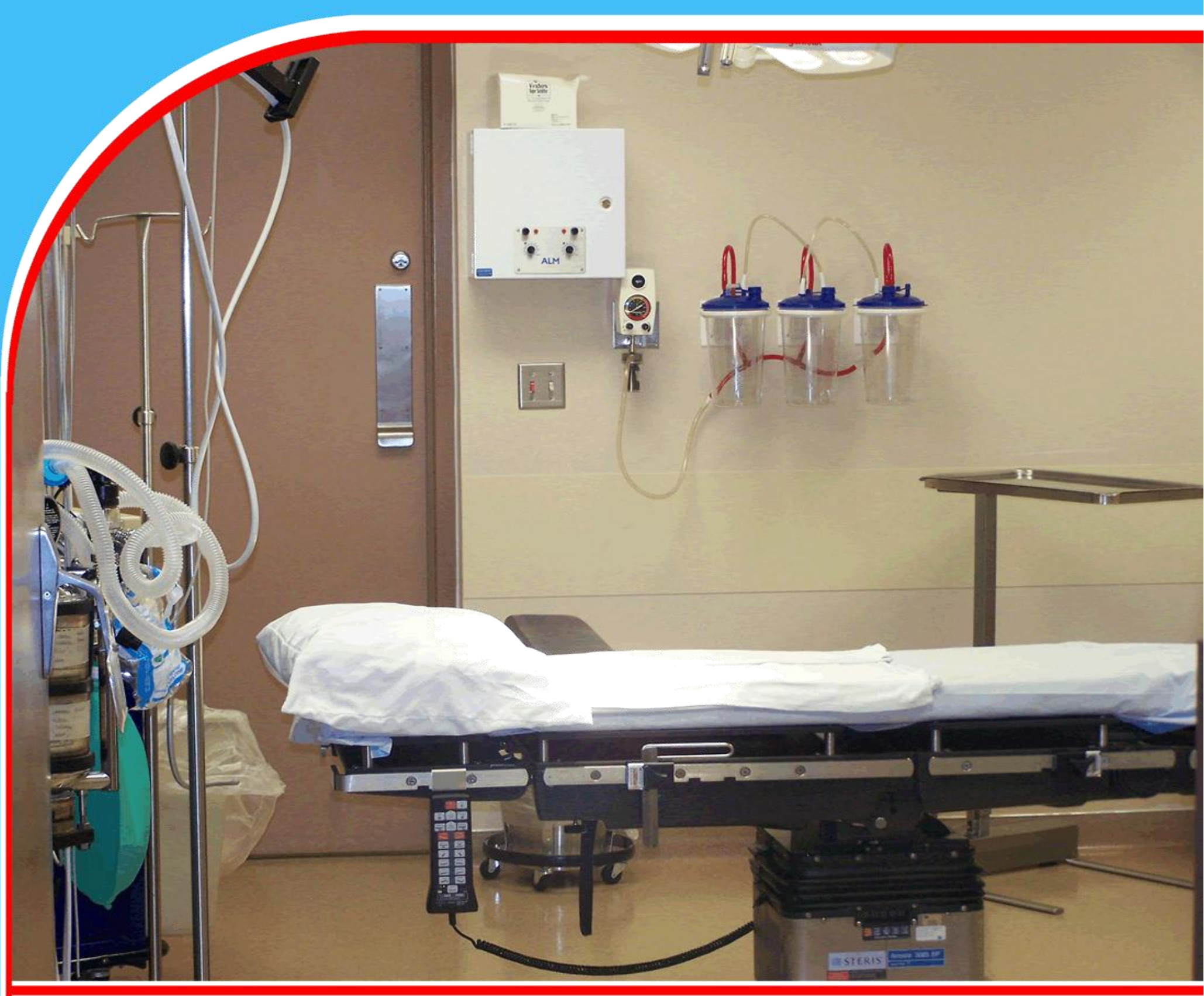

Features of various posterior cranial fossa tumors on Magnetic Resonance Imaging and their correlation with histopathological diagnosis Faiz Mohammed

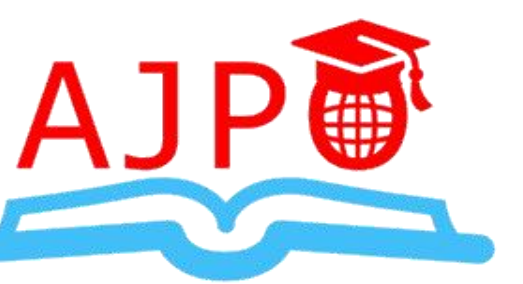




\title{
Features of various posterior cranial fossa tumors on Magnetic Resonance Imaging and their correlation with histopathological diagnosis
}

\section{Faiz Mohammed}

Department of radio-diagnosis, K.G. Hospital, Coimbatore, India

\begin{abstract}
Purpose: To evaluate the features of various posterior cranial fossa tumors on Magnetic Resonance Imaging and correlate the MR diagnosis with histopathological diagnosis.

Methodology: The MRI evaluation of patients clinically presenting with nonspecific symptoms of raised intracranial pressure was done. The MR imaging protocol included: T1W, T2W, FLAIR and post Gd T1W FS. The MRI diagnosis was correlated with histopathological diagnosis. Descriptive statistical analysis was carried out in the present study.

Findings: Histopathological confirmation was obtained in 38 of 46 cases. The eight patients in whom histopathologic examination was not obtained included four cases of metastasis and four cases of pontine glioma. In metastases, the primary was known. In pontine gliomas the characteristic MR findings were present and due to increased frequency of complications the risky biopsy in this area was not performed.

Unique contribution to theory, practice and policy (recommendations): The MR imaging diagnosis was found to correlate with the histopathologic diagnosis in most instances. MR imaging was found to be highly sensitive in evaluating tumor related complications. MRI showed the full extent of the tumor and involvement of the surrounding tissues, thus helping in the management of tumors. Hence it is recommended to perform MRI in cases of increased intracranial hypertension to make early and accurate diagnosis possible and hence improve the patient management.
\end{abstract}




\section{INTRODUCTION}

Tumors in the posterior fossa are considered critical brain lesions. This is, primarily, because of the limited space within the posterior fossa and the potential involvement of vital brain stem nuclei. Some patients should undergo an emergency operation, especially if they present with acute symptoms of brain stem involvement or herniation. Common posterior fossa brain tumors include juvenile pilocytic astrocytoma (JPA), medulloblastoma (MB), ependymoma and brainstem glioma. Less frequently, atypical teratoid/ rhabdoid tumor (ATRT), hemangioblastoma (HB), dermoids, schwannoma of the VIIIth cranial nerve, cerebellar gangliocytoma, meningioma, high grade glioma, and metastatic lesions are encountered. ${ }^{1}$

Worldwide the commonest pediatric posterior fossa tumors are medulloblastoma (20\%), astrocytoma (15\%), brainstem gliomas $(15 \%)$ and epedymomas $(8 \%)$ in that order. ${ }^{2}$ When these tumors acquire a bigger size and involve the deeper brain tissue, prognosis is less favorable $e^{3,4,5}$. Medulloblastoma is the commonest primary neuroectodermal tumour and also the most common solid childhood tumour. Five year survival rate is seen in $80 \%$ of patients when tumor is localized to posterior cranial fossa at time of diagnosis. ${ }^{6,7}$

The benign lesions like acoustic neuroma and schwannoma at cerebellopontine angle can also be life threatening due to their location adjacent to vital structures. The advancement in neuroradiological diagnostic techniques has resulted in detection of very small sized lesions which when managed effectively benefit the patient ${ }^{8}$. However, recurrence of benign lesions like meningiomas increases the morbidity and mortality anyways ${ }^{9}$. Topographically, posterior fossa tumors can be subdivided into extra-and intra-axial tumors. The latter group can be further split into brainstem, cerebellar, and fourth-ventricle tumors.

\section{Extra-axial Posterior Fossa Tumors:}

\section{Findings definitive of an extra axial lesion:}

1) CSF cleft between brain and the lesion

2) Vessels interposed between brain and lesion

3) Cortex between mass and edematous white matter

4) Dura (meninges) between (epidural) mass and brain .

\section{Findings suggestive of an extra axial lesion:}

1) Peripheral, broadly based along calvarium

2) Bone changes in overlying calvarium

3) Enhancement of adjacent meninges

4) Displacement of brain from skull.

\section{Intra axial posterior fossa tumors:}

- In contradistinction to extra axial tumors, intra axial tumors widen cortex and blur grey white interface rather than buckling the cortex.

- Intra axial tumors lack vascular/CSF/meningeal cleft. 
- Unlike extra axial tumors, they form an acute angle with the calvarium rather than being broad based towards it.

- They do not induce changes in the overlying calvarium.

- They do not displace brain away from skull but rather make it closely apposed to and deformed by the cortex. ${ }^{16}$

\section{MATERIALS AND METHODS}

This study was conducted in the department of Radio diagnosis, KG Hospital, Coimbatore, India. The researchers evaluated cases clinically suspected to have intracranial space occupying lesions. The diagnoses were confirmed histopathological. This was a prospective study.

\section{EQUIPMENT}

The equipment used was Siemens Magnetom Avanto 1.5 T.

\section{PATIENT POSITIONING}

The patient was placed supine on the MR Table and the head coil was positioned. A circularly polarized head coil was used for excitation and signal reception.

\section{CASE SELECTION}

The MRI evaluation of patients clinically presenting with nonspecific symptoms of raised intracranial pressure was done. Analysis of patients found to have posterior fossa tumor on MRI was performed. The patients were referred from neurology and neurosurgery departments of KG Hospital and some referred from outside hospitals. Exclusion criteria included all patients with general contraindications to MRI (ferromagnetic particles, cardiac pacemakers etc.) who were critically ill and those who were claustrophobic. Informed consent was obtained from patient /family member.

\section{MRI PROTOCOL}

The MR imaging protocol included: T1W, T2W, FLAIR and post Gd T1W FS. After localizer images were obtained, the axial images were tilted parallel to the corpus callosum. The sagittal images were planned on the axial sections. The coronal images were planned on the sagittal/axial sections. For T2W axial imaging, a turbo spin echo sequence was used with a repetition time (TR) of $3380 \mathrm{msec}$; echo time (TE) of $14.0 \mathrm{msec} ; 5 \mathrm{~mm}$ section thickness;30 mm intersection gap;150 flip angle;230 mm field of view;512 x512 matrix and imaging time of 3-minute 7seconds. For T1W sagittal imaging, a turbo spin echo sequence was used with a repetition time (TR) of 500 msec; echo time (TE) of $8.1 \mathrm{msec} ; 5 \mathrm{~mm}$ section thickness; $30 \mathrm{~mm}$ intersection gap; 90 flip angle; $230 \mathrm{~mm}$ field of view;512 x512 matrix and imaging time of 3 minute 55 seconds. For FLAIR axial imaging, a turbo spin echo sequence was used with a repetition time (TR) of $9000 \mathrm{msec}$; echo time (TE) of $87 \mathrm{msec} ; 5 \mathrm{~mm}$ section thickness;30 mm intersection gap;150 flip angle;230 mm field of view;512 x512 matrix and imaging time of 4 minute 32 seconds. For T2W coronal imaging, a turbo spin echo sequence was used with a repetition time (TR) of $3850 \mathrm{msec}$; echo time (TE) of $114 \mathrm{msec} ; 5 \mathrm{~mm}$ section thickness;30 mm intersection gap;150 flip angle;210 mm field of view;512 x512 matrix and imaging time of 2 minute 39 seconds. For post contrast imaging, Magnevist - Dimeglumine gadopentetate $(469 \mathrm{mg} / \mathrm{ml}$ ) was used in a dose of $0.2 \mathrm{mmol} / \mathrm{Kg}$ body weight. Following contrast administration, 2D T1W fat suppressed sequence was performed at 
2 minutes and 10minutes with a repetition time (TR) of $652 \mathrm{msec}$; echo time (TE) of $8.1 \mathrm{msec} ; 5$ $\mathrm{mm}$ section thickness; $30 \mathrm{~mm}$ intersection gap; $90^{\circ}$ flip angle; $230 \mathrm{~mm}$ field of view; $460 \times 512$ matrix and imaging time of 2 minute 30 seconds.

\section{STATISTICAL METHODS}

Descriptive statistical analysis was carried out in the present study. Results on continuous measurements were presented on Mean SD (Min-Max) and results on categorical measurements were presented in Number (\%). Significance was assessed at $5 \%$ level of significance. Analysis of variance (ANOVA) was used to find the significance of study parameters between three or more groups of patients, Student t test (two tailed, independent) was used to find the significance of study parameters on continuous scale between two groups, Chi-square/2x2, 2x3, 2x4 Fisher Exact test were used to find the significance of study parameters on categorical scale between two groups. $90 \%$ Confidence Interval was computed to find the significant features. Confidence Interval with a lower limit more than $50 \%$ was associated with statistical significance.

\section{Fisher Exact test}

Let there exist two such variables $X$ and $Y$, with $m$ and $n$ observed states, respectively. Now form

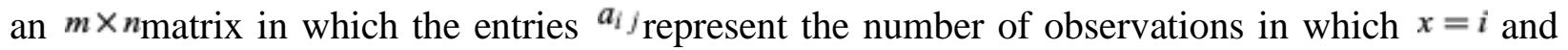
$y=j$. Calculate the row and column sums $R_{i}$ and $C_{j}$, respectively, and the total sum of the matrix. $N=\sum_{i} R_{i}=\sum_{j} C_{j}$

Then calculate the conditional probability of getting the actual matrix given the particular row and column sums, given by

$P_{\text {eutoff }}=\frac{\left(R_{1} ! R_{2} ! \cdots R_{m} !\right)\left(C_{1} ! C_{2} ! \cdots C_{n} !\right)}{N ! \prod_{l j} a_{l j} !}$,

The above formula is a multivariate generalization of the hyper geometric probability function

The sum of the probabilities of "unusual" tables gives the Fisher Exact test probability

\section{Analysis of Variance: $F$ test for $K$ Population means}

Objective: To test the hypothesis that K samples from K Populations with the same mean.

Limitations: It is assumed that populations are normally distributed and have equal variance. It is also assumed that samples are independent of each other.

Method. Let the $\mathrm{j}^{\text {th }}$ sample contain $\mathrm{n}_{\mathrm{j}}$ elements $(\mathrm{j}=1,2, \ldots \mathrm{K})$. Then the total number of elements is

$$
\begin{aligned}
& N=\sum n j \quad x . j=\sum \frac{x i j}{n j} \\
& S_{1}{ }^{2}=\frac{\sum \sum_{i=1}^{n 1}(x 1-\overline{x \cdot j})^{2}}{N-K} \quad S_{2}{ }^{2}=\frac{\sum_{i=1}^{n 1} n j(\overline{x \cdot j}-\bar{x} .)^{2}}{K-1}
\end{aligned}
$$

$\mathrm{F}=\mathrm{S}_{2}{ }^{2} / \mathrm{S}_{1}{ }^{2} \quad$ which follows $\mathrm{F}$ distribution $(\mathrm{K}-1, \mathrm{~N}-\mathrm{K})$ 


\section{Chi-Square Test}

$$
\chi^{2}=\frac{\sum(O i-E i)^{2}}{E i}, \text { Where Oi is observed frequency and Ei is Expected frequency }
$$

\section{Student $t$ test (Two tailed, independent)}

$$
\begin{aligned}
& t=\frac{\left(\bar{x}_{1}-\bar{x}_{2}\right)-\left(\mu_{1}-\mu_{2}\right)}{\sqrt{s^{2}(1 / n 1+1 / n 2)}} \\
& \text { Where } s^{2}=\frac{(n 1-1) \sum_{i=1}^{n 1}(x 1-\bar{x} 1)^{2}+(n 2-1) \sum_{i=1}^{n 2}(x 2-\bar{x} 2)^{2}}{n 1+n 2-2}
\end{aligned}
$$

\section{$5.90 \%$ Confidence Interval}

$$
\mathrm{P} \pm 1.645^{*} \mathrm{SE}(\mathrm{P}) \text {, Where SE }(\mathrm{P}) \text { is the Standard error of proportion }=\mathrm{P}^{*} \mathrm{Q} / \sqrt{ } \mathrm{n}
$$

\section{Diagnostic statistics}

\begin{tabular}{|l|l|l|l|l|l|}
\hline & Disease & & & & \\
\hline Test & Present & $\mathbf{n}$ & Absent & $\mathbf{n}$ & Total \\
\hline Positive & True Positive & $a$ & False Positive & $c$ & $a+c$ \\
\hline Negative & False Negative & $b$ & True Negative & $d$ & $b+d$ \\
\hline Total & & $a+b$ & & $c+d$ & \\
\hline
\end{tabular}

\section{Table 1: Diagnostic statistics}

The following statistics can be defined:

- Sensitivity: Probability that a test result will be positive when the disease is present (true positive rate, expressed as a percentage $).=a /(a+b)$.

- Specificity: Probability that a test result will be negative when the disease is not present (true negative rate, expressed as a percentage $).=\mathrm{d} /(\mathrm{c}+\mathrm{d})$.

- Positive predictive value: Probability that the disease is present when the test is positive (expressed as a percentage).$=\mathrm{a} /(\mathrm{a}+\mathrm{c})$.

- Negative predictive value: Probability that the disease is not present when the test is negative (expressed as a percentage).$=\mathrm{d} /(\mathrm{b}+\mathrm{d})$.

- Accuracy is the sum of true positive and true negative divided by number of cases.

\section{Diagnostic values based on Accuracy}

0.9-1.0 Excellent test

\section{8-1.9 Good test \\ 0.7-0.8 Fair test}




\section{6-0.7 Poor test \\ 0.5-0.6 Fail}

\section{Significant figures}

+ Suggestive significance ( $\mathrm{P}$ value: $0.05<\mathrm{P}<0.10)$

* Significant (P value: $0.01<\mathrm{P} 0.05)$

** Strongly significant (P value: P0.01)

\section{STATISTICAL SOFTWARE}

The Statistical software namely SPSS 13.0, Stata 8.0, MedCalc 9.0.1 and Systat 11.0 were used for the analysis of the data and Microsoft Word and Excel have been used to generate graphs, tables etc.

\section{RESULTS}

Analysis of the pattern of appearance of various posterior fossa tumors on magnetic resonance imaging was performed in the study. The MR diagnosis was correlated with histopathologic diagnosis.

\section{AGE AND GENDER DISTRIBUTION:}

\begin{tabular}{|l|c|c|c|c|}
\hline \multicolumn{4}{c}{ Age Distribution } \\
\multicolumn{1}{|c|}{ Age } & Male & Female & Total & $(\%)$ \\
\hline$<=16$ years (children) & 10 & 3 & 13 & $27 \%$ \\
\hline$>16$ (adults) & 11 & 22 & 33 & $69 \%$ \\
\hline Total & 21 & 25 & 46 & $96 \%$ \\
\hline
\end{tabular}

Table 2: Age distribution

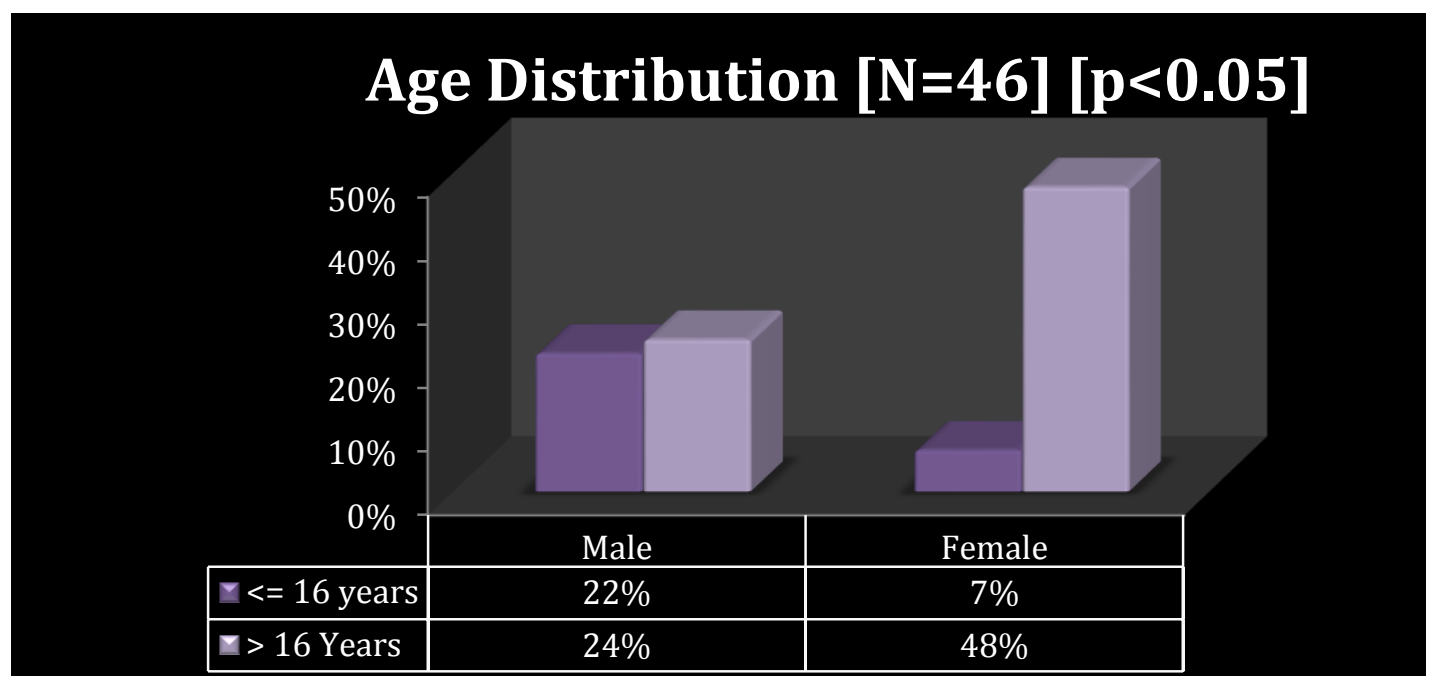

Figure 1: Age distribution 


\section{Sex Distribution}

\begin{tabular}{|c|c|c|c|}
\hline Age & Range & Mean +/- SD & p value \\
\hline Overall [N=46] & $2-75$ & $39+/-24$ & \\
\hline Male [n=21] & $2-75$ & $28+/-23$ & p $<0.05$ \\
\hline Female [n=25] & $2-75$ & $48+/-22$ & \\
\hline
\end{tabular}

Table 3: Sex distribution

\section{Sex Distribution [N=46]}

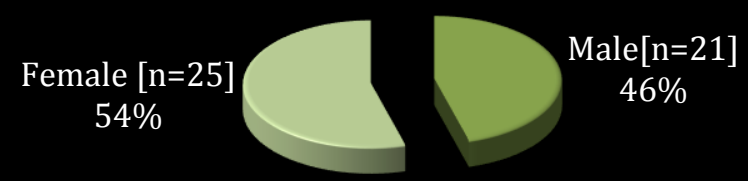

\section{Figure 2: Sex distribution}

Age range of patients in the study was two to seventy-five years. There are $13(27 \%)$ children (16years or less) and 33 (69\%) adults (more than 16 years). Overall female predominance (male: female $=1: 1.19)$ is seen $(54 \%)$. However, among children there is male $(77 \%)$ predominance (male: female=3.33:1 ). 
American Journal of Health, Medicine and Nursing Practice

\section{IMAGING DIAGNOSIS:}

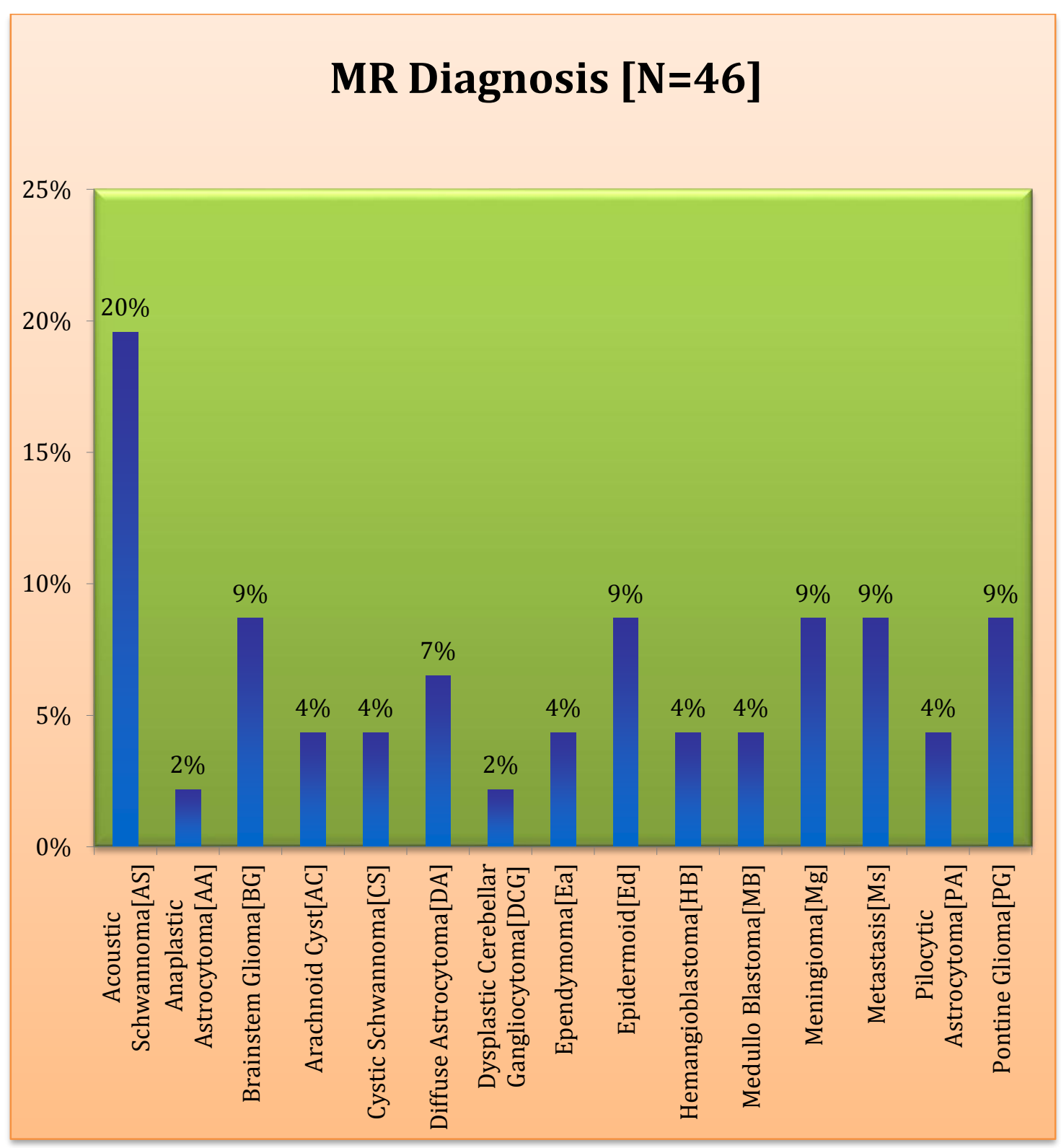

Figure 3: MR diagnosis 


\section{MR Diagnosis [ $\mathrm{N}=46]$}

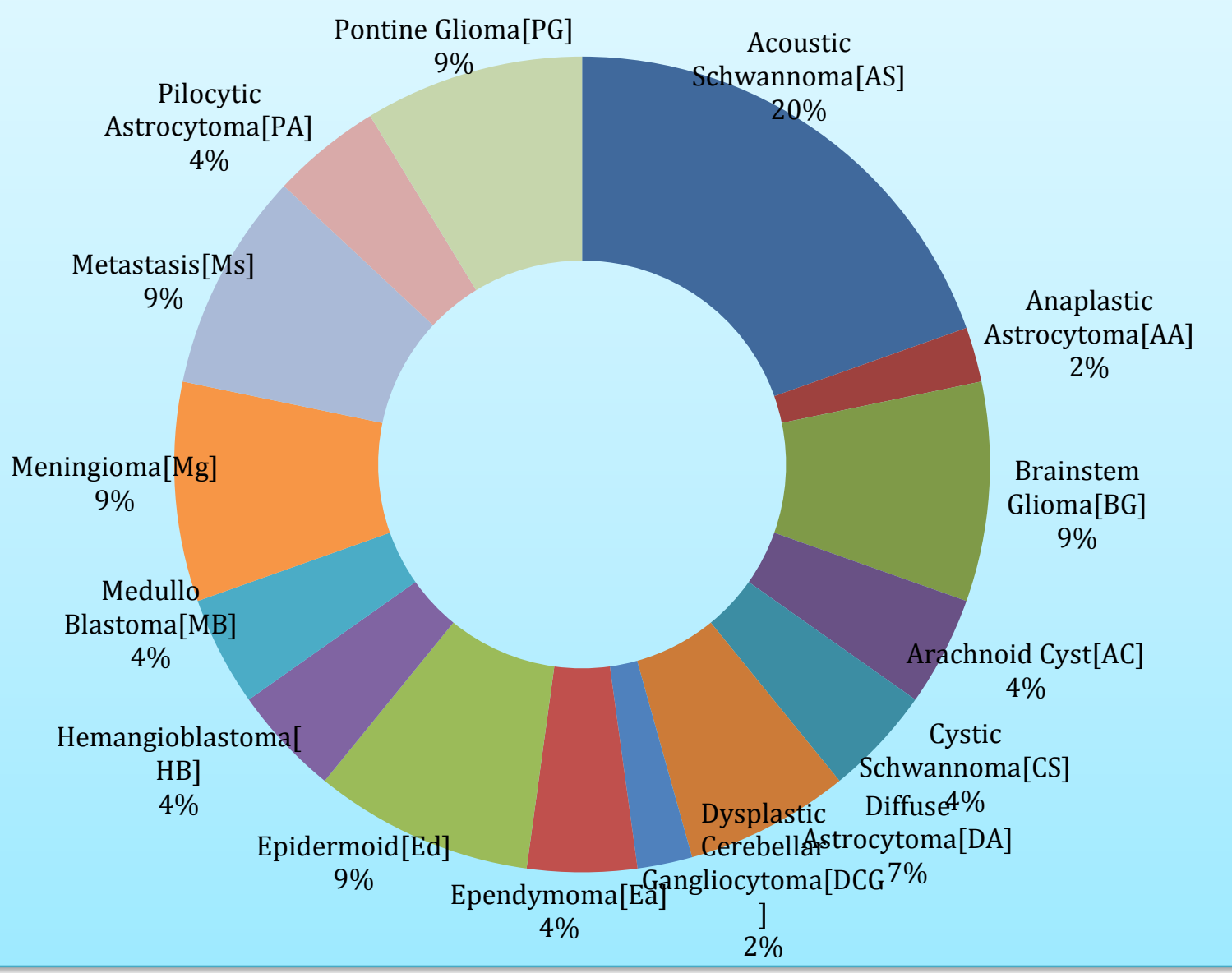

Figure 4: MR diagnosis

Most common tumor was acoustic schwannoma (AS-20\%). There was equal incidence (9\%) of brainstem glioma (BG), epidermoid (Ed), meningioma $(\mathrm{Mg})$, metastasis (Ms) and pontine glioma (PG). This was followed by equal incidence (4\%) of arachnoid cyst (AC), cystic schwannoma (CS), ependymoma (Ea), hemangioblastoma (HB), medulloblastoma (MB) and pilocytic astrocytoma (PA). Least common (2\% each) were anaplastic astrocytoma (AA) and dysplastic cerebellar gangliocytoma (DCG). 


\section{AGE WISE DISTRIBUTION OF TUMORS:}

\begin{tabular}{|c|c|c|c|c|}
\hline \multicolumn{4}{|c}{ Age wise distribution of tumors } \\
\hline Diagnosis & \multicolumn{2}{|c|}{ Age } & Total & $\mathbf{( \% )}$ \\
\hline Acoustic Schwannoma & $\mathbf{0}$ & $\mathbf{1 6}$ & & \\
\hline Anaplastic Astrocytoma & $\mathbf{0}$ & $\mathbf{1}$ & $\mathbf{9}$ & $\mathbf{2 0 \%}$ \\
\hline Brainstem Glioma & $\mathbf{3}$ & $\mathbf{1}$ & $\mathbf{4}$ & $\mathbf{2 \%}$ \\
\hline Arachnoid Cyst & $\mathbf{0}$ & $\mathbf{2}$ & $\mathbf{2}$ & $\mathbf{4 \%}$ \\
\hline Cystic Schwannoma & $\mathbf{0}$ & $\mathbf{2}$ & $\mathbf{2}$ & $\mathbf{4 \%}$ \\
\hline Diffuse Astrocytoma & $\mathbf{1}$ & $\mathbf{2}$ & $\mathbf{3}$ & $\mathbf{7 \%}$ \\
\hline Dysplastic Cerebellar Gangliocytoma & $\mathbf{0}$ & $\mathbf{1}$ & $\mathbf{1}$ & $\mathbf{2 \%}$ \\
\hline Ependymoma & $\mathbf{2}$ & $\mathbf{0}$ & $\mathbf{2}$ & $\mathbf{4 \%}$ \\
\hline Epidermoid & $\mathbf{0}$ & $\mathbf{4}$ & $\mathbf{4}$ & $\mathbf{9 \%}$ \\
\hline Hemangioblastoma & $\mathbf{0}$ & $\mathbf{2}$ & $\mathbf{2}$ & $\mathbf{4 \%}$ \\
\hline Medulloblastoma & $\mathbf{1}$ & $\mathbf{1}$ & $\mathbf{2}$ & $\mathbf{4 \%}$ \\
\hline Meningioma & $\mathbf{0}$ & $\mathbf{4}$ & $\mathbf{4}$ & $\mathbf{9 \%}$ \\
\hline Metastasis & $\mathbf{0}$ & $\mathbf{4}$ & $\mathbf{4}$ & $\mathbf{9 \%}$ \\
\hline Pilocytic Astrocytoma & $\mathbf{2}$ & $\mathbf{0}$ & $\mathbf{2}$ & $\mathbf{4 \%}$ \\
\hline Pontine Glioma & $\mathbf{4}$ & $\mathbf{0}$ & $\mathbf{4}$ & $\mathbf{9 \%}$ \\
\hline TOTAL & $\mathbf{1 3}$ & $\mathbf{3 3}$ & $\mathbf{4 6}$ & $\mathbf{1 0 0 \%}$ \\
\hline (\%) & $\mathbf{2 8 \%}$ & $\mathbf{7 2 \%}$ & $\mathbf{9 6 \%}$ & \\
\hline
\end{tabular}

Table 4: Age wise distribution of tumors

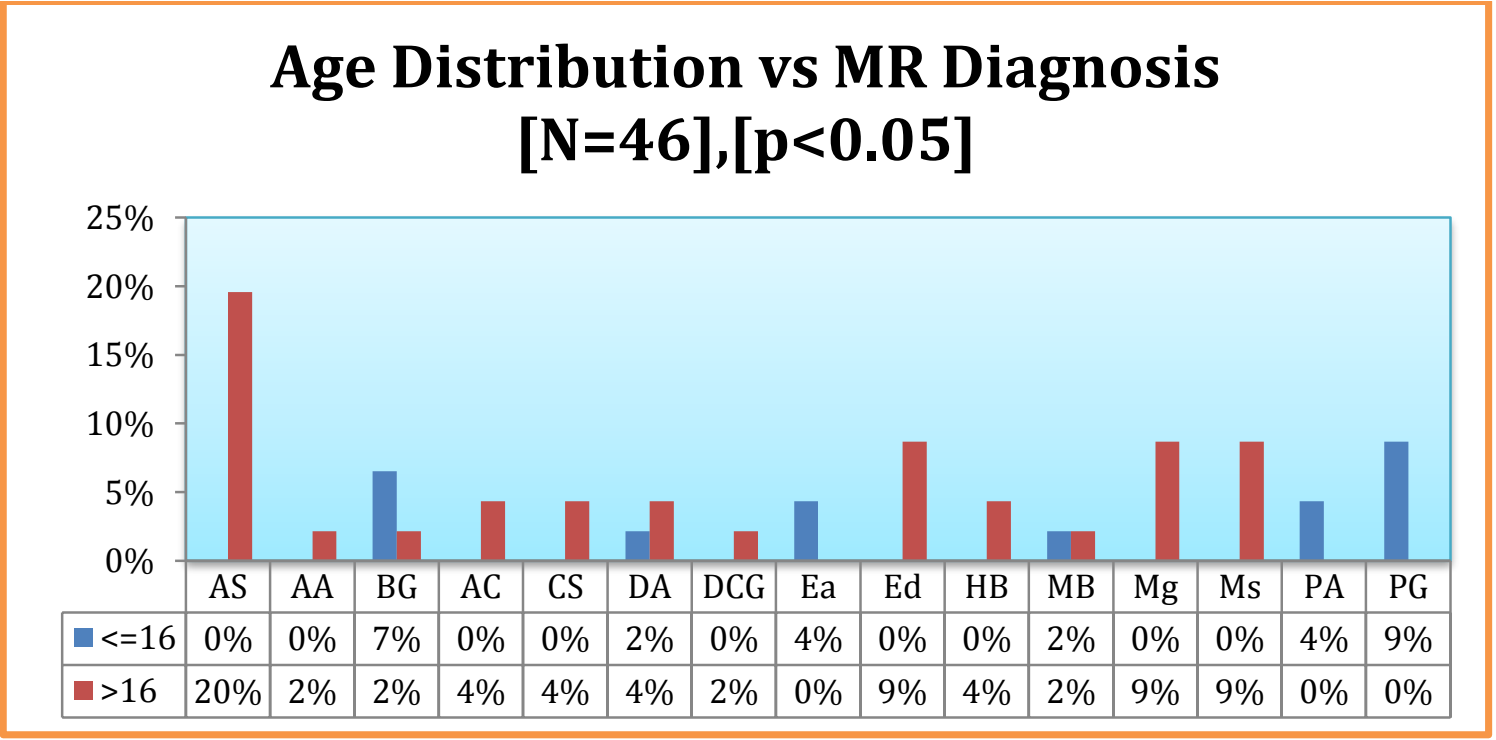

Figure 5: Age wise distribution of tumors 
Most of the patients were adults (72\%). Acoustic Schwannoma was most common tumor among adults. Metastasis (9\%), epidermoid (9\%), meningioma (9\%) and hemangioblastoma (4\%), were predominantly seen in adults. Pilocytoma (9\%), Ependymoma (9\%), medulloblastoma (9\%) and pontine glioma $(9 \%)$ were predominantly seen in children.

\section{GENDER WISE TUMOR DISTRIBUTION:}

\begin{tabular}{|c|c|c|c|c|}
\hline \multicolumn{5}{|c|}{ Sex vs MR Diagnosis } \\
\hline \multirow[t]{2}{*}{ Diagnosis } & \multicolumn{2}{|c|}{ Sex } & \multirow[t]{2}{*}{ Total } & \multirow[t]{2}{*}{$(\%)$} \\
\hline & Male & Female & & \\
\hline Acoustic Schwannoma & 3 & 6 & 9 & $20 \%$ \\
\hline Anaplastic Astrocytoma & 1 & 0 & 1 & $2 \%$ \\
\hline Brainstem Glioma & 3 & 1 & 4 & $9 \%$ \\
\hline Arachnoid Cyst & 2 & 0 & 2 & $4 \%$ \\
\hline Cystic Schwannoma & 0 & 2 & 2 & $4 \%$ \\
\hline Diffuse Astrocytoma & 3 & 0 & 3 & $7 \%$ \\
\hline Dysplastic Cerebellar Gangliocytoma & 0 & 1 & 1 & $2 \%$ \\
\hline Ependymoma & 0 & 2 & 2 & $4 \%$ \\
\hline Epidermoid & 0 & 4 & 4 & $9 \%$ \\
\hline Hemangioblastoma & 1 & 1 & 2 & $4 \%$ \\
\hline Medulloblastoma & 1 & 1 & 2 & $4 \%$ \\
\hline Meningioma & 0 & 4 & 4 & $9 \%$ \\
\hline Metastasis & 1 & 3 & 4 & $9 \%$ \\
\hline Pilocytic Astrocytoma & 2 & 0 & 2 & $4 \%$ \\
\hline Pontine Glioma & 4 & 0 & 4 & $9 \%$ \\
\hline TOTAL & 21 & 25 & 46 & $100 \%$ \\
\hline$(\%)$ & $46 \%$ & $54 \%$ & $100 \%$ & \\
\hline
\end{tabular}

Table 5: Gender wise distribution of tumors 


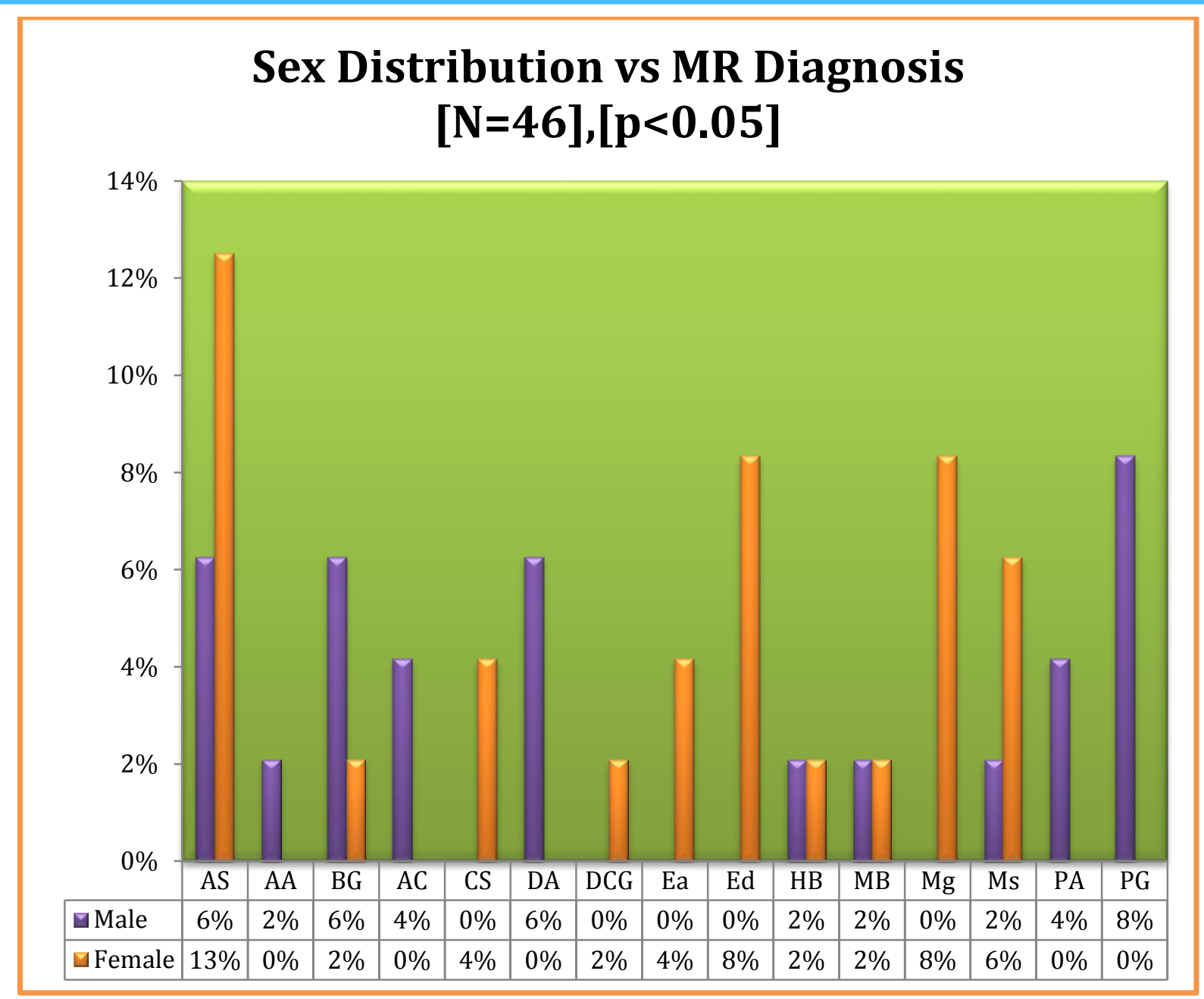

Figure 6: Gender wise distribution of tumors

Acoustic schwannoma is the most common tumor in females (20\%). Pontine glioma is the most common tumor in males (9\%). 


\section{LOCATION WISE IMAGING DIAGNOSIS:}

\begin{tabular}{|c|c|c|c|c|}
\hline \multicolumn{5}{|c|}{ MR Diagnosis vs Location } \\
\hline \multirow[t]{2}{*}{ Diagnosis } & \multicolumn{2}{|c|}{ Location } & \multirow[t]{2}{*}{ Total } & \multirow[t]{2}{*}{$(\%)$} \\
\hline & Extra Axial & Intra Axial & & \\
\hline Acoustic Schwannoma & 9 & 0 & 9 & $20 \%$ \\
\hline Anaplastic Astrocytoma & 0 & 1 & 1 & $2 \%$ \\
\hline Brainstem Glioma & 0 & 4 & 4 & $9 \%$ \\
\hline Arachnoid Cyst & 2 & 0 & 2 & $4 \%$ \\
\hline Cystic Schwannoma & 2 & 0 & 2 & $4 \%$ \\
\hline Diffuse Astrocytoma & 0 & 3 & 3 & $7 \%$ \\
\hline $\begin{array}{l}\text { Dysplastic Cerebellar } \\
\text { Gangliocytoma }\end{array}$ & 1 & 0 & 1 & $2 \%$ \\
\hline Ependymoma & 0 & 2 & 2 & $4 \%$ \\
\hline Epidermoid & 4 & 0 & 4 & $9 \%$ \\
\hline Hemangioblastoma & 0 & 2 & 2 & $4 \%$ \\
\hline Medulloblastoma & 0 & 2 & 2 & $4 \%$ \\
\hline Meningioma & 3 & 1 & 4 & $9 \%$ \\
\hline Metastasis & 0 & 4 & 4 & $9 \%$ \\
\hline Pilocytic Astrocytoma & 0 & 2 & 2 & $4 \%$ \\
\hline Pontine Glioma & 0 & 4 & 4 & $9 \%$ \\
\hline TOTAL & 21 & 25 & 46 & $100 \%$ \\
\hline$(\%)$ & $46 \%$ & $54 \%$ & $100 \%$ & \\
\hline
\end{tabular}

Table 6: Location wise distribution of tumors 


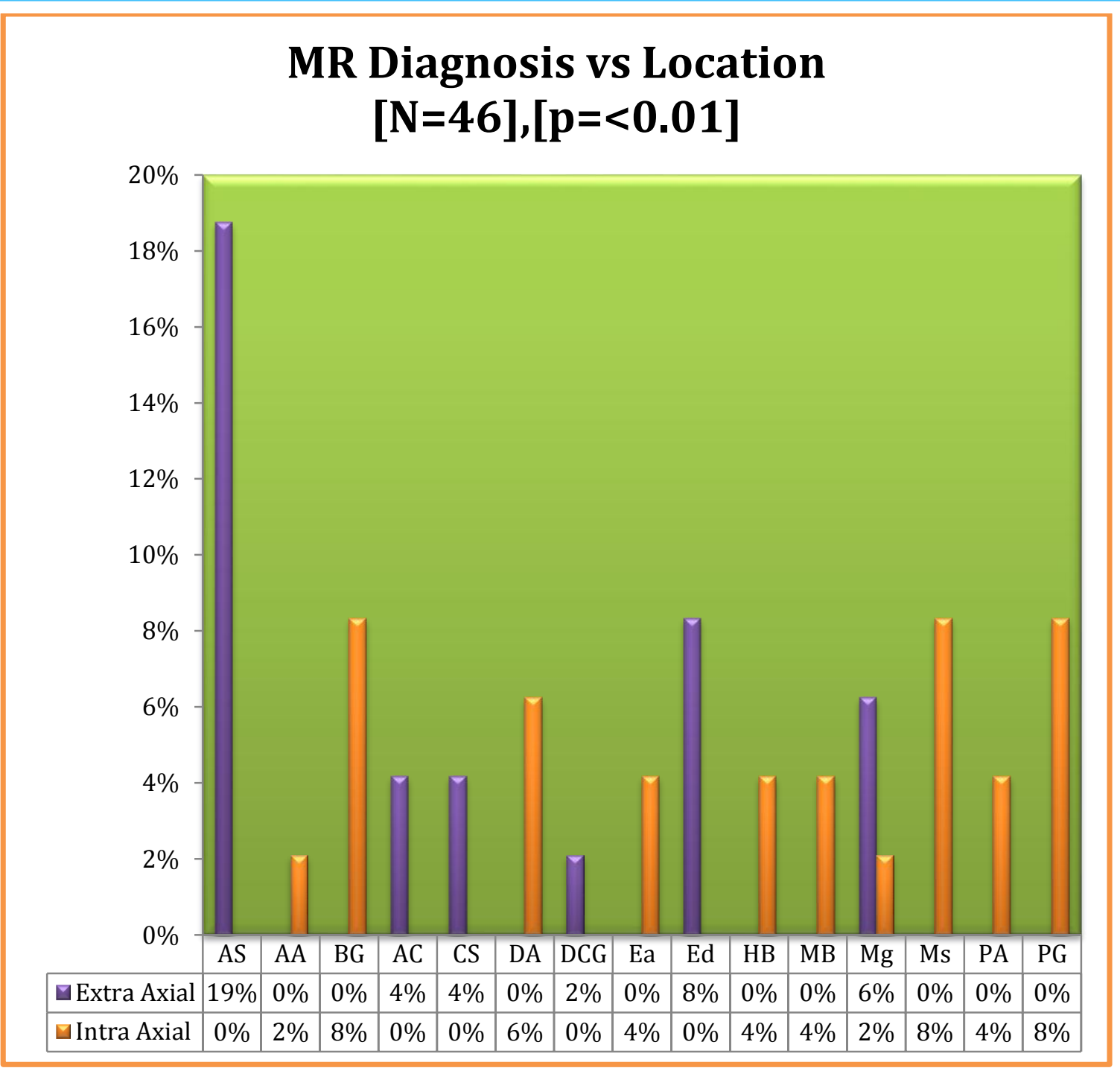

\section{Figure 7: Location wise distribution of tumors}

Tumors in 21 patients were extra axial (46\%). These include: Schwannoma (20\%), meningioma (9\%), epidermoid (9\%) and arachnoid cyst (4\%). The remaining 25 patients $(54 \%)$ had intra axial tumors which included: Metastasis $(9 \%)$, pilocytic astrocytoma (4\%), ependymoma (4\%), pontine glioma (9\%), hemangioblastoma (4\%), medulloblastoma (4\%), diffuse astrocytoma (7\%), brainstem glioma (9\%), anaplastic astrocytoma (2\%), dysplastic cerebellar gangliocytoma (2\%). 


\section{PLANE OF THE TUMORS}

Plane vs diagnosis

\begin{tabular}{|l|l|}
\hline Plane & Total No. of patients \\
\hline Vermis & 1 \\
\hline Brainstem & 6 \\
\hline $4^{\text {th }}$ ventricle & 1 \\
\hline Cerebellum & 8 \\
\hline CP angle cistern & 12 \\
\hline Tentorial & 2 \\
\hline
\end{tabular}

\section{Table 7: Plane wise distribution of tumors}

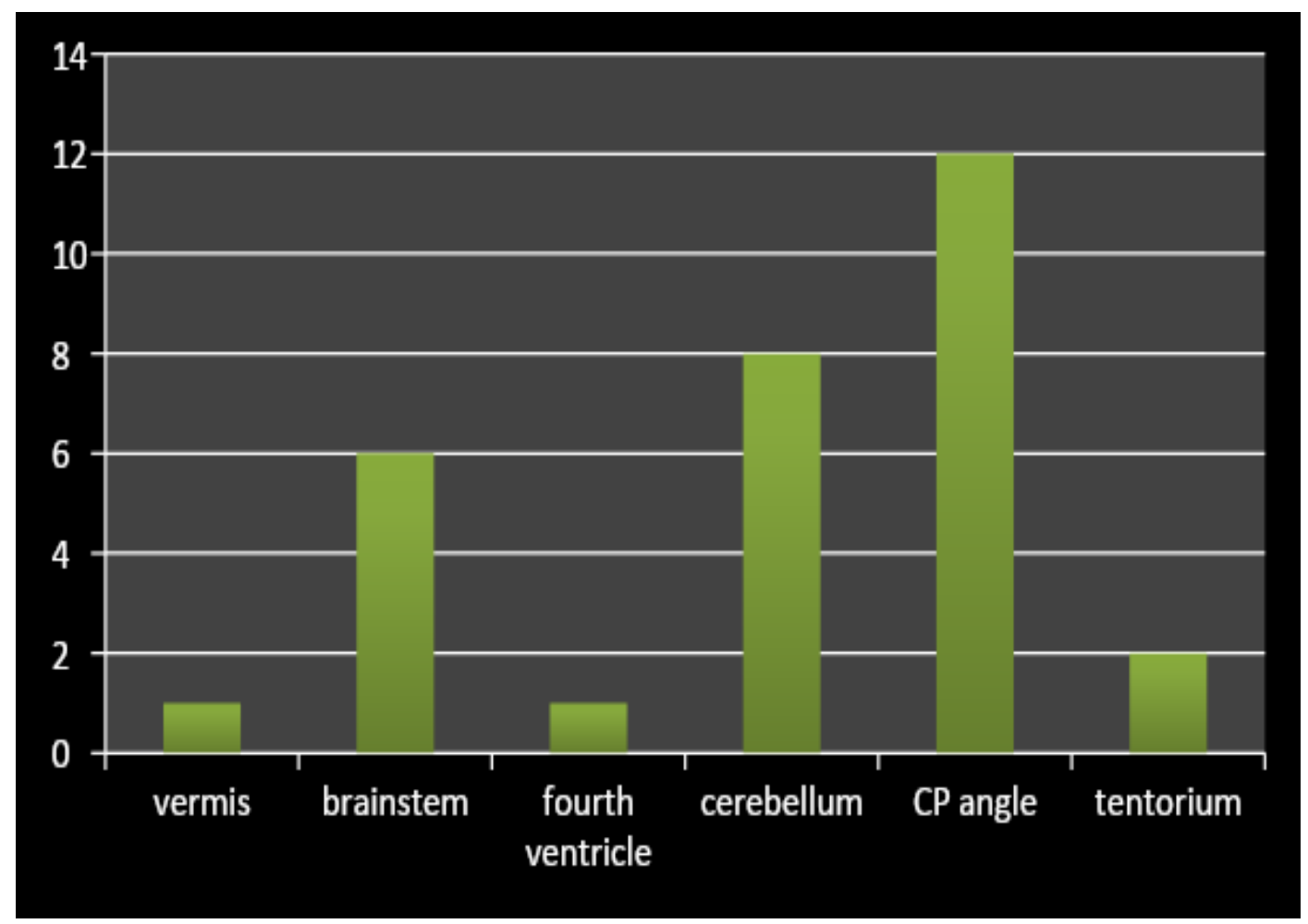

Figure 8: Plane wise distribution of tumors

Most of the tumors were located in the CP angle (12) and least in the Vermis (1) and Fourth Ventricle (1). 
VII. MR PATTERN ON T1 WEIGHTED IMAGE:

\begin{tabular}{|c|c|c|c|c|}
\hline \multicolumn{5}{|c|}{ MR Diagnosis vs T1 Weighted Image } \\
\hline \multirow[t]{2}{*}{ Diagnosis } & \multicolumn{2}{|c|}{ T1 Weighted Image } & \multirow[t]{2}{*}{ Total } & \multirow[t]{2}{*}{$(\%)$} \\
\hline & Hypointense & Isointense & & \\
\hline Acoustic Schwannoma & 9 & 0 & 9 & $20 \%$ \\
\hline Anaplastic Astrocytoma & 1 & 0 & 1 & $2 \%$ \\
\hline Brainstem Glioma & 4 & 0 & 4 & $9 \%$ \\
\hline Arachnoid Cyst & 2 & 0 & 2 & $4 \%$ \\
\hline Cystic Schwannoma & 2 & 0 & 2 & $4 \%$ \\
\hline Diffuse Astrocytoma & 2 & 1 & 3 & $7 \%$ \\
\hline $\begin{array}{l}\text { Dysplastic Cerebellar } \\
\text { Gangliocytoma }\end{array}$ & 1 & 0 & 1 & $2 \%$ \\
\hline Ependymoma & 2 & 0 & 2 & $4 \%$ \\
\hline Epidermoid & 4 & 0 & 4 & $9 \%$ \\
\hline Hemangioblastoma & 1 & 1 & 2 & $4 \%$ \\
\hline Medulloblastoma & 1 & 1 & 2 & $4 \%$ \\
\hline Meningioma & 1 & 3 & 4 & $9 \%$ \\
\hline Metastasis & 4 & 0 & 4 & $9 \%$ \\
\hline Pilocytic Astrocytoma & 2 & 0 & 2 & $4 \%$ \\
\hline Pontine Glioma & 4 & 0 & 4 & $9 \%$ \\
\hline TOTAL & 40 & 6 & 46 & $100 \%$ \\
\hline (\%) & $87 \%$ & $13 \%$ & $100 \%$ & \\
\hline
\end{tabular}

Table 8: MR pattern on $T 1$ weighted image 


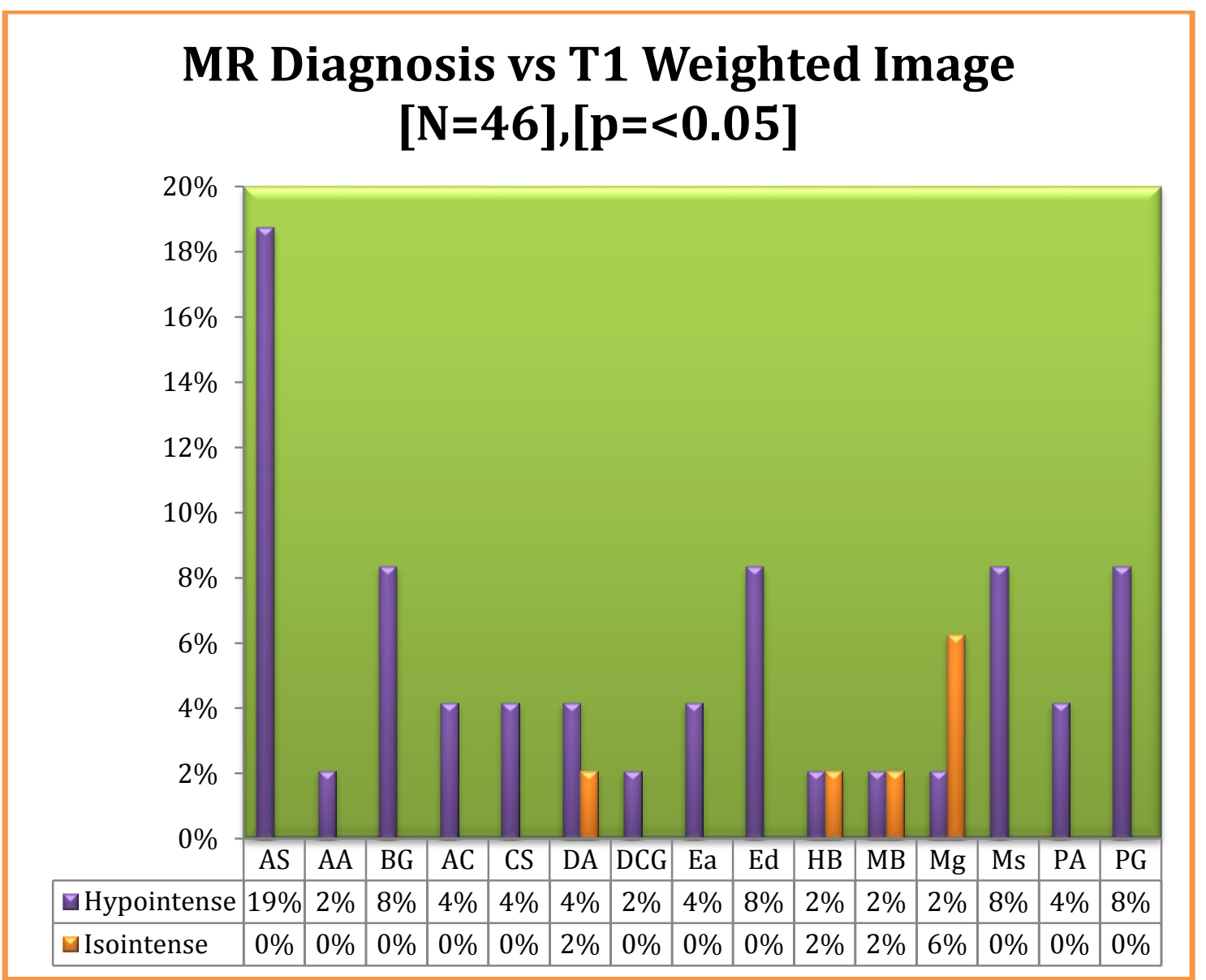

Figure 9: MR pattern on T1 weighted image

Most of the cases were hypointense on T1WI (87\%) while 13\% were isointense. These include three cases of meningioma and one case each of diffuse astrocytoma, hemangioblastoma and medulloblastoma. 
VIII. MR PATTERN ON T2 WEIGHTED IMAGE:

\begin{tabular}{|c|c|c|c|c|c|}
\hline \multicolumn{6}{|c|}{ Diagnosis vs T2 Weighted Image } \\
\hline \multirow[t]{2}{*}{ Diagnosis } & \multicolumn{3}{|c|}{ T2 Weighted Image } & \multirow[t]{2}{*}{ Total } & \multirow[t]{2}{*}{$(\%)$} \\
\hline & Hyperintense & Heterogenous & Isointense & & \\
\hline Acoustic Schwannoma & 0 & 9 & 0 & 9 & $20 \%$ \\
\hline Anaplastic Astrocytoma & 1 & 0 & 0 & 1 & $2 \%$ \\
\hline Brainstem Glioma & 4 & 0 & 0 & 4 & $9 \%$ \\
\hline Arachnoid Cyst & 2 & 0 & 0 & 2 & $4 \%$ \\
\hline Cystic Schwannoma & 2 & 0 & 0 & 2 & $4 \%$ \\
\hline Diffuse Astrocytoma & 3 & 0 & 0 & 3 & $7 \%$ \\
\hline $\begin{array}{l}\text { Dysplastic Cerebellar } \\
\text { Gangliocytoma }\end{array}$ & 1 & 0 & 0 & 1 & $2 \%$ \\
\hline Ependymoma & 2 & 0 & 0 & 2 & $4 \%$ \\
\hline Epidermoid & 4 & 0 & 0 & 4 & $9 \%$ \\
\hline Hemangioblastoma & 2 & 0 & 0 & 2 & $4 \%$ \\
\hline Medulloblastoma & 0 & 0 & 2 & 2 & $4 \%$ \\
\hline Meningioma & 0 & 0 & 4 & 4 & $9 \%$ \\
\hline Metastasis & 4 & 0 & 0 & 4 & $9 \%$ \\
\hline Pilocytic Astrocytoma & 2 & 0 & 0 & 2 & $4 \%$ \\
\hline Pontine Glioma & 4 & 0 & 0 & 4 & $9 \%$ \\
\hline TOTAL & 31 & 9 & 6 & 46 & $100 \%$ \\
\hline (\%) & $65 \%$ & $19 \%$ & $13 \%$ & $96 \%$ & \\
\hline
\end{tabular}

Table 9: MR pattern on T2 weighted image 


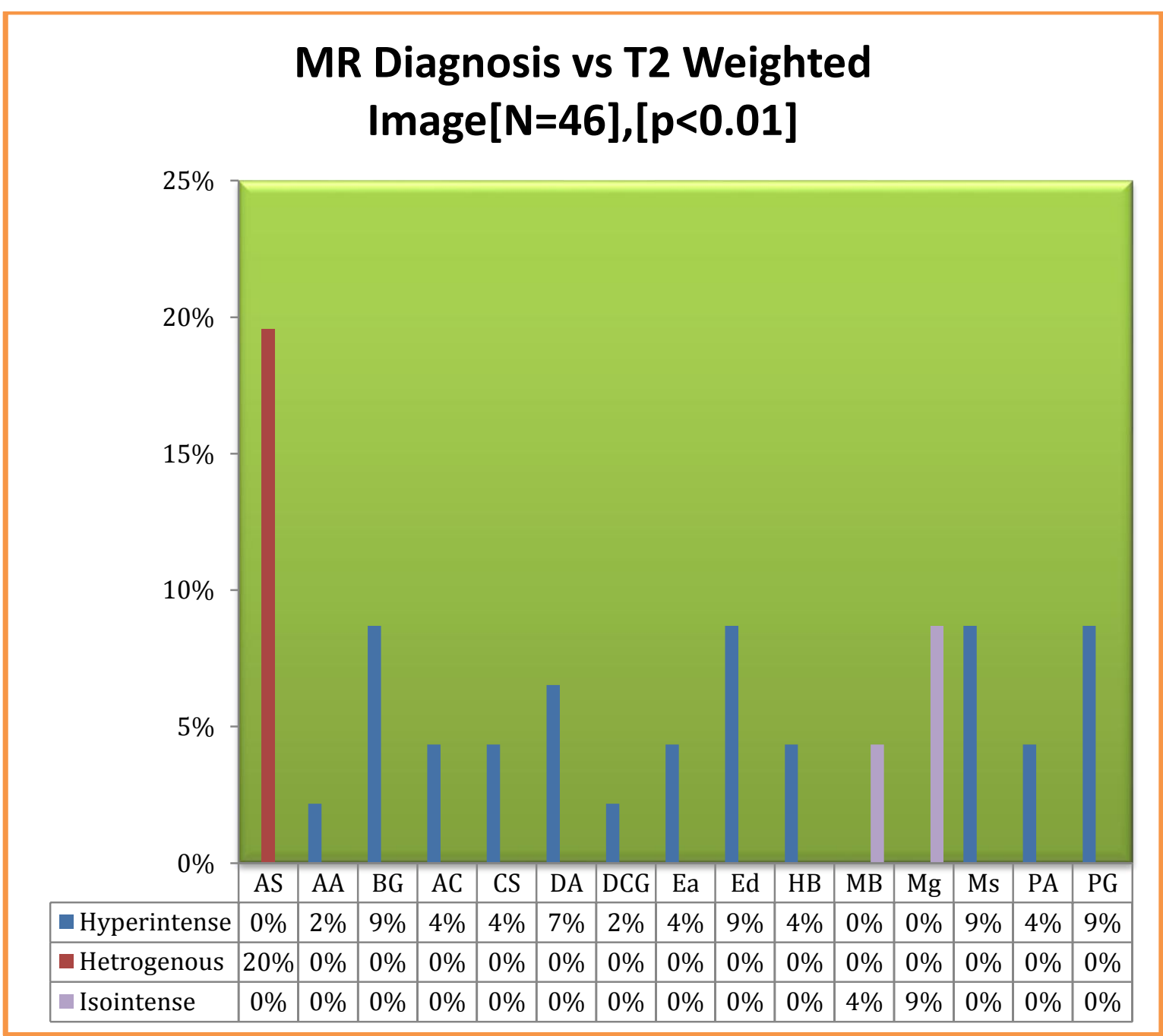

Figure 10: MR pattern on T2 weighted image

Most of the tumors were predominantly hyperintense on T2WI (65\%) whereas $19 \%$ showed a heterogeneous signal which mainly included Schwannoma. 13\% were isointense. These included four cases of meningioma and two cases of medulloblastoma. 


\section{MR PATTERN ON FLUID ATTENUATED INVERSION RECOVERY (FLAIR) IMAGE:}

MR Diagnosis vs Fluid Attenuated Inversion Recovery Image

\begin{tabular}{|c|c|c|c|c|c|c|}
\hline \multirow[t]{2}{*}{ Diagnosis } & \multicolumn{4}{|c|}{ FLAIR } & \multirow[t]{2}{*}{ Total } & \multirow[t]{2}{*}{$(\%)$} \\
\hline & Нуро & Hyper & Hetero & Iso & & \\
\hline Acoustic Schwannoma & 0 & 0 & 9 & 0 & 9 & $20 \%$ \\
\hline Anaplastic Astrocytoma & 0 & 1 & 0 & 0 & 1 & $2 \%$ \\
\hline Brainstem Glioma & 0 & 4 & 0 & 0 & 4 & $9 \%$ \\
\hline Arachnoid Cyst & 2 & 0 & 0 & 0 & 2 & $4 \%$ \\
\hline Cystic Schwannoma & 2 & 0 & 0 & 0 & 2 & $4 \%$ \\
\hline Diffuse Astrocytoma & 0 & 3 & 0 & 0 & 3 & $7 \%$ \\
\hline $\begin{array}{l}\text { Dysplastic Cerebellar } \\
\text { Gangliocytoma }\end{array}$ & 1 & 0 & 0 & 0 & 1 & $2 \%$ \\
\hline Ependymoma & 0 & 2 & 0 & 0 & 2 & $4 \%$ \\
\hline Epidermoid & 0 & 4 & 0 & 0 & 4 & $9 \%$ \\
\hline Hemangioblastoma & 1 & 1 & 0 & 0 & 2 & $4 \%$ \\
\hline Medulloblastoma & 0 & 1 & 0 & 1 & 2 & $4 \%$ \\
\hline Meningioma & 0 & 0 & 0 & 4 & 4 & $9 \%$ \\
\hline Metastasis & 0 & 4 & 0 & 0 & 4 & $9 \%$ \\
\hline Pilocytic Astrocytoma & 2 & 0 & 0 & 0 & 2 & $4 \%$ \\
\hline Pontine Glioma & 0 & 3 & 0 & 1 & 4 & $9 \%$ \\
\hline TOTAL & 8 & 23 & 9 & 6 & 46 & $100 \%$ \\
\hline$(\%)$ & $17 \%$ & $50 \%$ & $20 \%$ & $13 \%$ & $100 \%$ & \\
\hline
\end{tabular}

Table 10: MR pattern on FLAIR 


\section{MR Diagnosis vs FLAIR[ $\mathrm{N}=46],[\mathrm{p}<0.01]$}

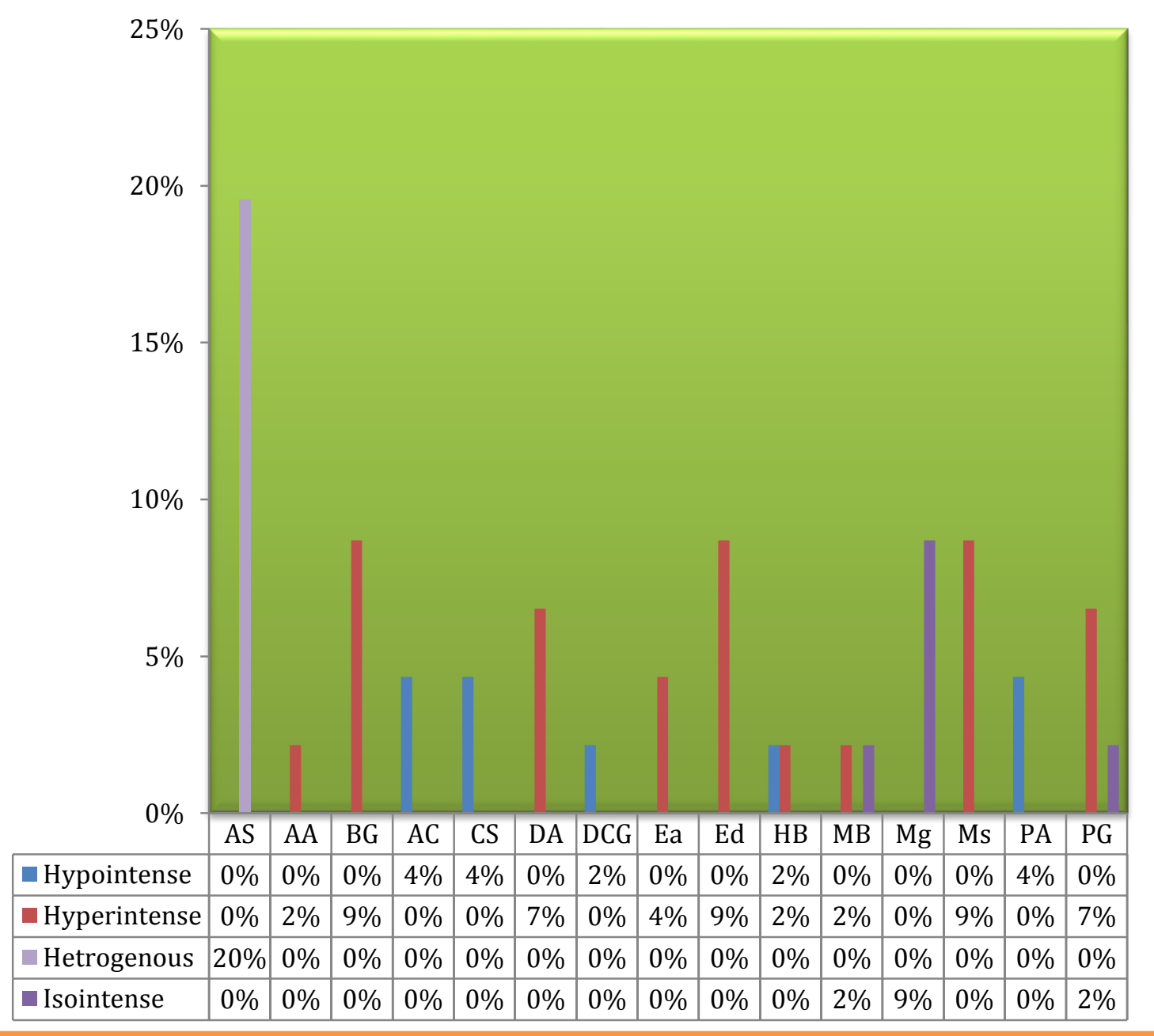

Figure 11: MR pattern on FLAIR

Most of the tumors were hyperintense on FLAIR (50\%). 20\% showed heterogenous signal which mainly included schwannoma. 17\% were hypointense including two cases each of arachnoid cyst, cystic schwannoma, pilocytic astrocytoma and one case each of dysplastic cerebellar gangliocytoma and hemangioblastoma. 


\section{MR PATTERN ON POSTCONTRAST IMAGES:}

\begin{tabular}{|c|c|c|c|c|c|c|}
\hline \multicolumn{7}{|c|}{ MR Diagnosis vs Post Contrast } \\
\hline \multirow[t]{2}{*}{ Diagnosis } & \multicolumn{4}{|c|}{ Post Contrast } & \multirow[t]{2}{*}{ Total } & \multirow[t]{2}{*}{$(\%)$} \\
\hline & Moderate & Intense & Peripheral & $\begin{array}{c}\text { No } \\
\text { Enhancement }\end{array}$ & & \\
\hline Acoustic Schwannoma & 0 & 8 & 0 & 1 & 9 & $20 \%$ \\
\hline Anaplastic Astrocytoma & 1 & 0 & 0 & 0 & 1 & $2 \%$ \\
\hline Brainstem Glioma & 2 & 0 & 0 & 2 & 4 & $9 \%$ \\
\hline Arachnoid Cyst & 0 & 0 & 0 & 2 & 2 & $4 \%$ \\
\hline Cystic Schwannoma & 0 & 0 & 2 & 0 & 2 & $4 \%$ \\
\hline Diffuse Astrocytoma & 0 & 0 & 0 & 3 & 3 & $7 \%$ \\
\hline $\begin{array}{l}\text { Dysplastic Cerebellar } \\
\text { Gangliocytoma }\end{array}$ & 0 & 0 & 0 & 1 & 1 & $2 \%$ \\
\hline Ependymoma & 2 & 0 & 0 & 0 & 2 & $4 \%$ \\
\hline Epidermoid & 0 & 0 & 0 & 4 & 4 & $9 \%$ \\
\hline Hemangioblastoma & 0 & 1 & 0 & 1 & 2 & $4 \%$ \\
\hline Medulloblastoma & 0 & 2 & 0 & 0 & 2 & $4 \%$ \\
\hline Meningioma & 0 & 4 & 0 & 0 & 4 & $9 \%$ \\
\hline Metastasis & 0 & 4 & 0 & 0 & 4 & $9 \%$ \\
\hline Pilocytic Astrocytoma & 0 & 2 & 0 & 0 & 2 & $4 \%$ \\
\hline Pontine Glioma & 0 & 0 & 0 & 4 & 4 & $9 \%$ \\
\hline TOTAL & 5 & 21 & 2 & 18 & 46 & $100 \%$ \\
\hline$(\%)$ & $11 \%$ & $46 \%$ & $4 \%$ & $39 \%$ & $100 \%$ & \\
\hline
\end{tabular}

Table 11: MR pattern on post contrast images 


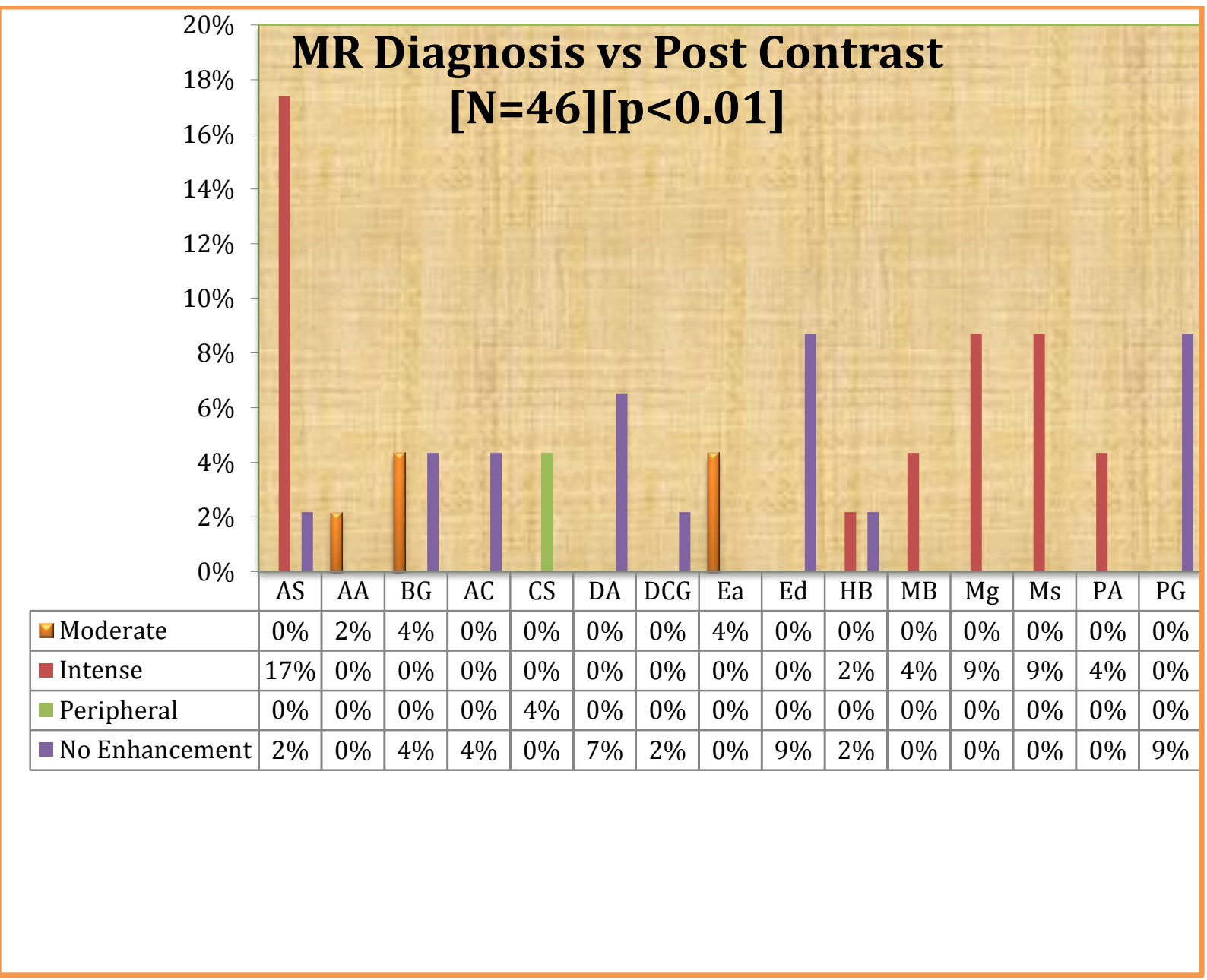

Figure 12: MR pattern on post contrast images

Most of the tumors showed intense enhancement (46\%). In 39\% there was no appreciable enhancement. Moderate enhancement was seen in 11\%. Least common pattern was peripheral enhancement (4\%).

\section{CORRELATION OF MR DIAGNOSIS WITH HISTOPATHOLOGIC DIAGNOSIS.}

Histopathological confirmation was obtained in 38 of 46 cases. The eight patients in whom histopathologic examination was not obtained included four cases of metastasis and four cases of pontine glioma. In metastases, the primary was known. In pontine gliomas the characteristic MR findings were present and due to increased frequency of complications the risky biopsy in this area was not performed. 


\begin{tabular}{|c|c|c|c|c|}
\hline \multicolumn{5}{|c|}{ MR Diagnosis vs HP } \\
\hline \multirow[t]{2}{*}{ MR Diagnosis } & \multicolumn{2}{|c|}{ HP } & \multirow[t]{2}{*}{ Total } & \multirow[t]{2}{*}{$(\%)$} \\
\hline & Positive & Negative & & \\
\hline Acoustic Schwannoma & 7 & 2 & 9 & $24 \%$ \\
\hline Anaplastic Astrocytoma & 1 & 0 & 1 & $3 \%$ \\
\hline Brainstem Glioma & 3 & 1 & 4 & $11 \%$ \\
\hline Arachnoid Cyst & 2 & 0 & 2 & $5 \%$ \\
\hline Cystic Schwannoma & 2 & 0 & 2 & $5 \%$ \\
\hline Diffuse Astrocytoma & 3 & 0 & 3 & $8 \%$ \\
\hline $\begin{array}{l}\text { Dysplastic Cerebellar } \\
\text { Gangliocytoma }\end{array}$ & 1 & 0 & 1 & $3 \%$ \\
\hline Ependymoma & 2 & 0 & 2 & $5 \%$ \\
\hline Epidermoid & 4 & 0 & 4 & $11 \%$ \\
\hline Hemangioblastoma & 2 & 0 & 2 & $5 \%$ \\
\hline Medulloblastoma & 2 & 0 & 2 & $5 \%$ \\
\hline Meningioma & 4 & 0 & 4 & $11 \%$ \\
\hline Pilocytic Astrocytoma & 2 & 0 & 2 & $5 \%$ \\
\hline TOTAL & 35 & 3 & 38 & $100 \%$ \\
\hline (\%) & $92 \%$ & $8 \%$ & $100 \%$ & \\
\hline
\end{tabular}

Table 12: Correlation of MR diagnosis with histopathologic diagnosis 


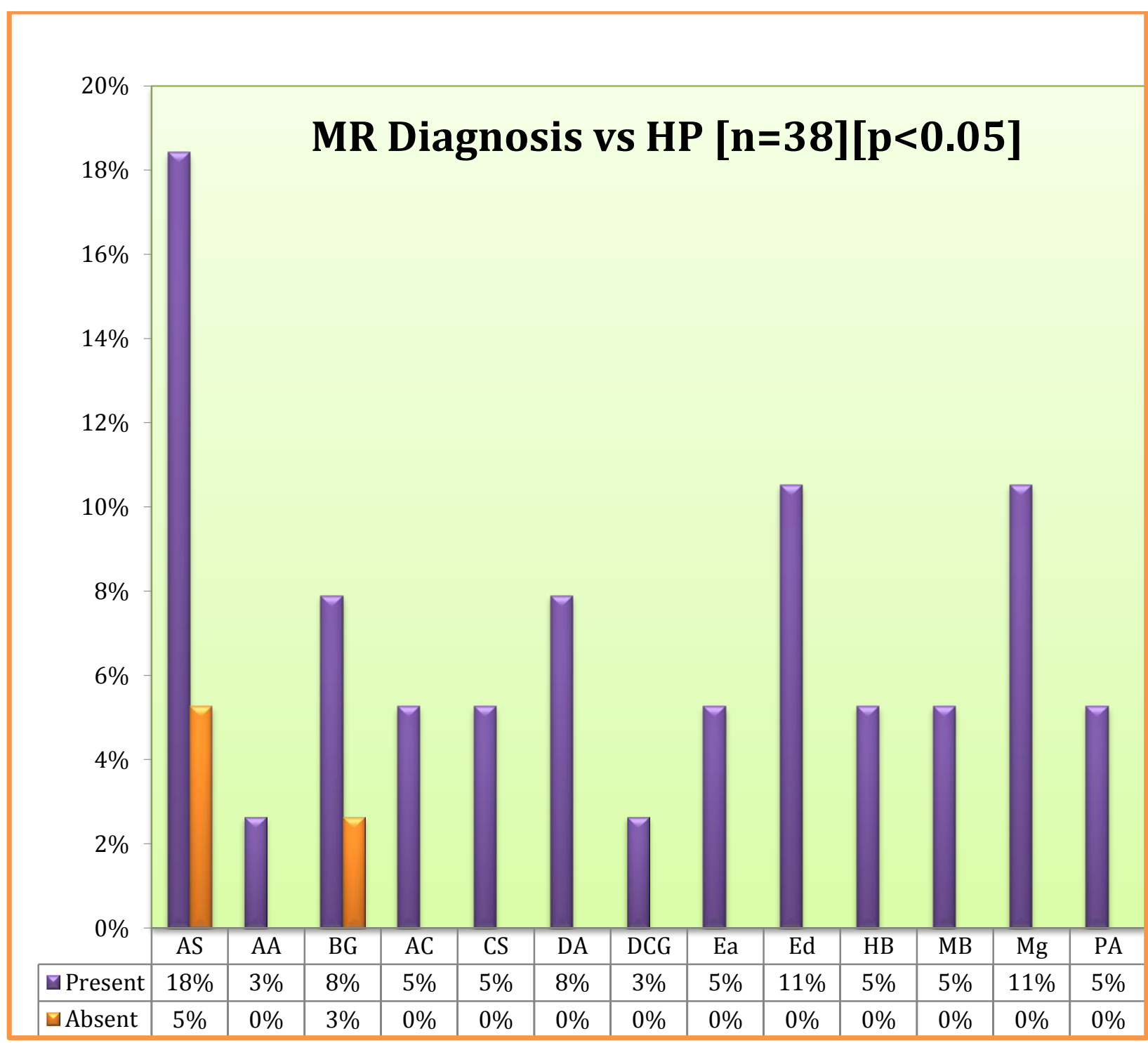

Figure 13: Correlation of MR diagnosis with histopathologic diagnosis

A correct diagnosis was reached by MRI in 35 of 38 patients, a positive predictive value of $92 \%$. 


\section{Correlation of MRI Diagnosis with HPE: an observation}

\begin{tabular}{|c|c|c|c|c|c|}
\hline Diagnosis & $\begin{array}{c}\text { True } \\
\text { Positive }\end{array}$ & $\begin{array}{l}\text { False } \\
\text { Positive }\end{array}$ & $\begin{array}{c}\text { False } \\
\text { Negative }\end{array}$ & $\begin{array}{c}\text { True } \\
\text { Negative }\end{array}$ & $\begin{array}{c}\text { Total number } \\
\text { of patients }\end{array}$ \\
\hline Acoustic Schwannoma & 7 & 2 & 0 & 29 & 38 \\
\hline Anaplastic Astrocytoma & 1 & 0 & 0 & 37 & 38 \\
\hline Brainstem Glioma & 3 & 1 & 0 & 34 & 38 \\
\hline Arachnoid Cyst & 2 & 0 & 0 & 36 & 38 \\
\hline Cystic Schwannoma & 2 & 0 & 0 & 36 & 38 \\
\hline Diffuse Astrocytoma & 3 & 0 & 0 & 35 & 38 \\
\hline $\begin{array}{l}\text { Dysplastic Cerebellar } \\
\text { Gangliocytoma }\end{array}$ & 1 & 0 & 0 & 37 & 38 \\
\hline Ependymoma & 2 & 0 & 0 & 36 & 38 \\
\hline Epidermoid & 4 & 0 & 0 & 34 & 38 \\
\hline Hemangioblastoma & 2 & 0 & 0 & 36 & 38 \\
\hline Medulloblastoma & 2 & 0 & 0 & 36 & 38 \\
\hline Meningioma & 4 & 0 & 0 & 34 & 38 \\
\hline Pilocytic Astrocytoma & 2 & 0 & 0 & 36 & 38 \\
\hline
\end{tabular}

Table 13: Correlation of MR diagnosis with HPE: an observation 


\begin{tabular}{|l|c|c|c|c|c|}
\hline \multicolumn{5}{|c|}{ Correlation of MRI Diagnosis with HPE: an Evaluation } \\
\hline \multicolumn{1}{|c|}{ Diagnosis } & Sensitivity & Specificity & PPV & NPV & Accuracy \\
\hline Acoustic Schwannoma & 100 & 94 & 78 & 100 & 75 \\
\hline Anaplastic Astrocytoma & 100 & 100 & 100 & 100 & 100 \\
\hline Brainstem Glioma & 100 & 97 & 75 & 100 & 97 \\
\hline Arachnoid Cyst & 100 & 100 & 100 & 100 & 100 \\
\hline Cystic Schwannoma & 100 & 100 & 100 & 100 & 100 \\
\hline Diffuse Astrocytoma & 100 & 100 & 100 & 100 & 100 \\
\hline $\begin{array}{l}\text { Dysplastic Cerebellar } \\
\text { Gangliocytoma }\end{array}$ & 100 & 100 & 100 & 100 & 100 \\
\hline Ependymoma & 100 & 100 & 100 & 100 & 100 \\
\hline Epidermoid & 100 & 100 & 100 & 100 & 100 \\
\hline Hemangioblastoma & 100 & 100 & 100 & 100 & 100 \\
\hline Medulloblastoma & 100 & 100 & 100 & 100 & 100 \\
\hline Meningioma & 100 & 100 & 100 & 100 & 100 \\
\hline Pilocytic Astrocytoma & & 100 & 100 & 100 \\
\hline S & 100 & & & \\
\hline
\end{tabular}

Table 14: Correlation of MR diagnosis with HPE: an evaluation

MRI was found to be $100 \%$ sensitive and specific in diagnosing all posterior fossa tumors except schwannoma and brainstem glioma. In cases of schwannoma, MRI was 100\% sensitive and had $100 \%$ negative predictive value. However, specificity was $94 \%$ and positive predictive value only $78 \%$, bringing down the accuracy to $75 \%$. In cases of brainstem glioma, MRI was $100 \%$ sensitive and had $100 \%$ negative predictive value. MR was also found to have a good specificity of $97 \%$. However, the positive predictive value was only $75 \%$. Overall, the accuracy of MR in diagnosing brainstem glioma was $97 \%$.

\section{DISCUSSION}

Common posterior fossa brain tumors include juvenile pilocytic astrocytoma (JPA), medulloblastoma (MB), ependymoma and brainstem glioma. Less frequently, atypical teratoid/rhabdoid tumor (ATRT), hemangioblastoma (HB), dermoids, schwannoma of the VIIIth 
American Journal of Health, Medicine and Nursing Practice

ISSN 2520-4017 (Online)

Vol.6, Issue 4, pp $40-88,2021$

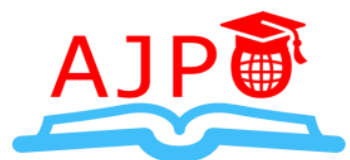

www.ajpojournals.org

cranial nerve, cerebellar gangliocytoma, meningioma, high grade glioma and metastatic lesions are encountered. ${ }^{1}$

Because these various tumors require very different treatment approaches and have significantly different natural histories and outcomes, an accurate and specific diagnosis is mandatory. Conventional MRI is essential for diagnosis as well as evaluation of location and extent of the tumor. Bilaniuk LT reported that in adults, the combination of clinical presentation and imaging results permit prediction of the histology of a posterior fossa tumor. MR imaging, which uses various pulse sequences and contrast material is the most sensitive and precise technique for the evaluation of patients who have symptoms referable to the posterior fossa. ${ }^{21}$

\section{PATIENT DEMOGRAPHICS}

MRI of 46 patients who presented with clinical features of intracranial space occupying lesions and found to have posterior fossa tumor on MR imaging were studied prospectively in our institution. Age range of patients in our study was two to 75 years. Most of the patients were adults (72\%). Acoustic Schwannoma was the most common tumor among adults. Metastasis (9\%), epidermoids (9\%), meningioma (9\%) and hemangioblastoma (4\%), were predominantly seen in adults. Among children (28\%), the most common tumor was pontine glioma(9\%). Pilocytoma (4\%), Ependymoma (9\%), medulloblastoma (9\%) are predominantly seen in children. Rehman et al studied 62 patients with posterior fossa tumors and found similar incidence. ${ }^{25}$ In our study, 25 patients were females and 21 were males. Overall there was slight female predominance with the male: female ratio of 1: 1.19. P.K Wanyoike et al found a similar trend in their study of 37 patients of posterior fossa tumors. ${ }^{26}$ However in the pediatric age group there were only three females and remaining 10 patients were males. Chang T et al studied 129 cases of posterior fossa tumors in childhood and reports similar findings. ${ }^{27}$

\section{MRI features}

MRI features evaluated include: number of lesions, location, morphology, mass effect and signal characteristics on T1W, T2W, FLAIR and post contrast images. The tumors were solitary in 42 cases. Four patients had multiple metastatic tumors. Tumors in 21 patients were extra axial (46\%). These included: Schwannoma (20\%), meningioma (9\%), epidermoid (9\%) and arachnoid cyst (4\%). The remaining 25 patients $(54 \%)$ had intra axial tumors which included: Metastasis $(9 \%)$, pilocytic astrocytoma (4\%), ependymoma (4\%), pontine glioma (9\%), hemangioblastoma (4\%), medulloblastoma (4\%), diffuse astrocytoma (7\%), brainstem glioma (9\%), anaplastic astrocytoma $(2 \%)$, dysplastic cerebellar gangliocytoma (2\%). The extraaxial tumors demonstrated $\mathrm{CSF} / \mathrm{vascular} / \mathrm{meningeal}$ cleft with rotated brainstem and buckling of cerebellum around the tumor. The intraaxial tumors on the contrary lacked CSF/vascular/meningeal cleft and involved and infiltrated the brainstem and cerebellum. These findings correspond to the description of Curnes JT. ${ }^{29}$ Most of the tumors were located in the CP angle (12) and least in the Vermis (1) and Fourth Ventricle (1). In 15 cases the tumors were solid, cystic in 7 cases and showed both solid and cystic components in 24 . Fourth ventricle was compressed in 25 patients with hydrocephalus in 20 patients. Brainstem was compressed or rotated in 11 patients. Cerebellar herniation was seen in 4 patients. There was no mass effect in the remaining 6 patients. The peritumoral oedema was of infiltrative type representing both vasogenic oedema and tumor cell infiltration. 


\section{Signal characteristics of each tumor are described below:}

Acoustic schwannoma: All of eight cases of acoustic schwannoma were centered at the internal auditory meatus. This finding was observed by Mulkens $\mathrm{TH}$ et $\mathrm{al}^{30}$ in their study on acoustic tumors. However, intracanalicular extension is not a specific feature of Schwannoma and can be seen with non-acoustic tumors as well. This finding correlates with that of Izyeka. ${ }^{31}$ All cases showed low signal on TIWI, mixed signal on T2WI / FLAIR and intense but heterogeneous enhancement. These findings are in agreement with those of Mulkens TH et al. ${ }^{30}$ Two cases of schwannoma with cystic change were seen. They were hypointense on $\mathrm{T} 1 \mathrm{~W}$, hyperintense on $\mathrm{T} 2 \mathrm{~W}$ and hypointense on FLAIR images. Peripheral enhancement was seen in both tumors. The findings correspond to those described by $\mathrm{M}$ Wackoh et al. ${ }^{32}$

Meningioma: There were four cases of meningioma in our study. All the patients were female. Two were broad based on the tentorium. All the four tumors were isointense on T1WI and were isointense to hypointense on T2W/FLAIR imaging. Similar findings were reported by Francesco Maiuri. ${ }^{33}$ All cases showed homogenous enhancement. Our findings were in agreement with those of Scott $\mathrm{W}^{34}$ who stated that meningiomas have a strong female predilection. Over half were isointense and one-third were hypointense to the cortex on T1WI. Nearly all meningiomas enhance rapidly and intensely.

"Dural tail sign" was demonstrated in two cases of tentorial based meningiomas as observed by Takeguchi T and Hadi Rokni-Yazdi. ${ }^{35}$ We had one case of intraparenchymal meningioma which was iso to hypointense on T1W and isointense to hyperintense on $\mathrm{T} 2 \mathrm{~W}$ and showed homogenous enhancement on contrast administration and these findings were same as reported by Carlos Majos. ${ }^{36}$ Magnetic resonance (MR) imaging reliably demonstrates typical features of vestibular schwannomas or meningiomas in the vast majority of mass lesions in the cerebellopontine angle (CPA). This finding correlates with that of Bonneville. ${ }^{37}$

Epidermoid cyst: Four cases of epidermoid cyst were seen. All were located in the CPA. All were hypointense on T1WI, hyperintense on T2WI and FLAIR. These findings are similar to those of Dutt SN et $\mathrm{al}^{38}$ and Wasenko JJ et al. ${ }^{39}$ In one case in which there was compression and displacement of the fourth ventricle, there was no hydrocephalus, similar to the study by Kallmes DF et al. $^{40}$

Arachnoid cyst: There were two cases of arachnoid cyst in our study. The intensity was similar to cerebrospinal fluid (CSF) on all the sequences. On FLAIR imaging it was completely suppressed (low signal). These findings were consistent with the study published by Dutt SN et al. ${ }^{38}$

Medulloblastoma: There were two cases of medulloblastoma in young children, filling the fourth ventricle arising from the Vermis (roof of fourth ventricle) which were isointense on T1WI and T2W/FLAIR imaging and showed heterogeneous enhancement on contrast administration. These findings are similar to those seen by Rodallec $\mathrm{M}$ et al. ${ }^{41}$

Ependymoma: Qiu SJ and Zhang XL ${ }^{42}$ reported MR imaging findings of 13 cases of intracranial ependymomas. The T1W images presented isointense or hypointense signals and T2W/FLAIR images showed inhomogeneous hyperintense signals. No edema occurred around these tumors and the contrast-enhanced scans presented inhomogeneous enhancement. There were two cases of fourth ventricular ependymoma in the present study with similar features and which were extending down through the foramen of Magendie. On the basis of differences in localization, 
enhancement and tumor growth in most cases a preoperative differential diagnosis between medulloblastomas and ependymomas is possible. Warmuth et al also made a similar conclusion. ${ }^{43}$

Cerebellar astrocytoma: There were six cerebellar astrocytomas in our study. Two were pilocytic astrocytoma, three were diffuse astrocytoma and one anaplastic astrocytoma. Juvenile pilocytic astrocytomas were well circumscribed round cystic lesions with solid nodule. The cystic component was hypointense on T1WI, hyperintense on T2WI and hypointense on FLAIR. The solid nodule was isointense to grey matter on T1WI, hyperintense to grey matter but hypointense to CSF on T2WI and hyperintense to grey matter on FLAIR. Intense postcontrast enhancement was seen in the solid nodule. Lee YY 1989, Coakley KJ et al 1995 and Koleller KK et al 2004 reviewed 37, 56 and 45 cases of juvenile pilocytic astrocytoma respectively. Their description of the MR pattern of the tumor was the same as in our study. They concluded that by using age of presentation, typical location, configuration, and enhancement patterns, the presurgical diagnosis of juvenile pilocytic astrocytoma can be made with a high index of confidence. ${ }^{44,45,46 .}$

Brain stem glioma: In our study group there were four cases of tectal glioma and four cases of pontine gliomas. All the patients were of younger age group and MRI morphological features were similar to those described by Zimmerman $\mathrm{RA}^{47}$. All the brain stem tumours located in the pons encased the basilar artery which was identified due to flow void.

Hemangioblastoma: There were two cases of hemangioblastoma located in the right cerebellar hemisphere. The tumours were cystic with enhancing mural nodule. They were hypointense on T1W, FLAIR imaging, and hyperintense on T2W imaging. This finding was similar to that noted by Lee SR et al. ${ }^{48}$

Metastasis: Four cases of metastasis were evaluated. In both the female patients the primary were proved cases of carcinoma of breast and in the male patients it was bronchogenic carcinoma. The lesions were multiple and located at grey white interface. They showed intense nodular and ring enhancement. These findings were similar to those reported by Zhou CW. ${ }^{49}$

\section{CORRELATION OF MRI DIAGNOSIS WITH HISTOPATHOLOGY:}

Histopathological confirmation was obtained in 38 of 46 cases. The eight patients in whom histopathologic examination was not obtained included four cases of metastasis and four cases of pontine glioma. In metastases, the primary was known. In pontine gliomas the characteristic MR findings were present and due to increased frequency of complications the risky biopsy in this area was not performed. A correct diagnosis was reached by MRI in 35 of 38 patients, a positive predictive value of $92 \%$. MRI was found to be $100 \%$ sensitive and specific in diagnosing all posterior fossa tumors except schwannoma and brainstem glioma. In cases of schwannoma, MRI was $100 \%$ sensitive and had $100 \%$ negative predictive value. However, specificity was $94 \%$ and positive predictive value only $78 \%$, bringing down the accuracy to $75 \%$. In cases of brainstem glioma, MRI was $100 \%$ sensitive and had $100 \%$ negative predictive value. MR was also found to have a good specificity of $97 \%$. However, the positive predictive value was only $75 \%$. Overall, the accuracy of MR in diagnosing brainstem glioma was $97 \%$. 


\section{ILLUSTRATIVE CASES}

\section{CASE - I}

A 24-year-old man presented with a long-standing occipital headache, progressive ataxia and blurred vision. He has been vomiting for 8-10 days. Fundoscopy revealed papilloedema. MRI was performed:

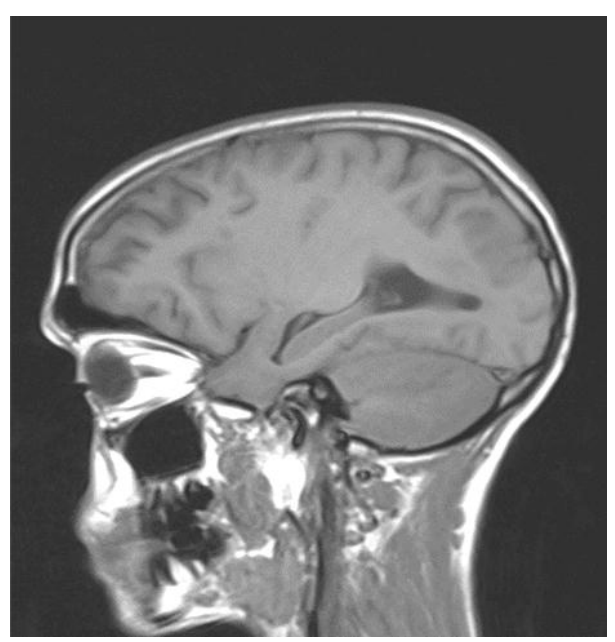

Figure 14: T1 weighted sagittal image: The lesion is difficult to visualize except subtle accentuation of cerebellar folia appearing iso to mildly hypointense.
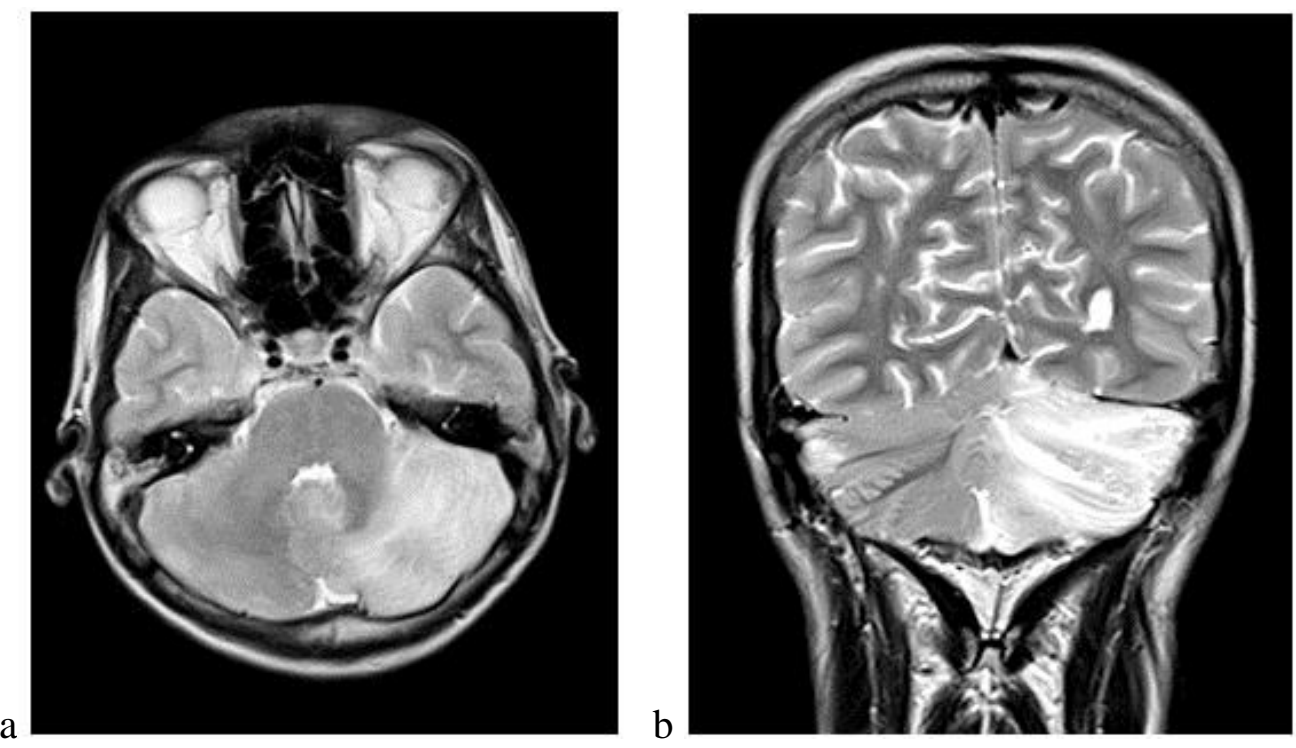

Figure 15a\&b: T2 weighted axial/coronal images: The lesion is predominantly hyperintense, with the typical alternate high- and normal-signal-intensity bands. 


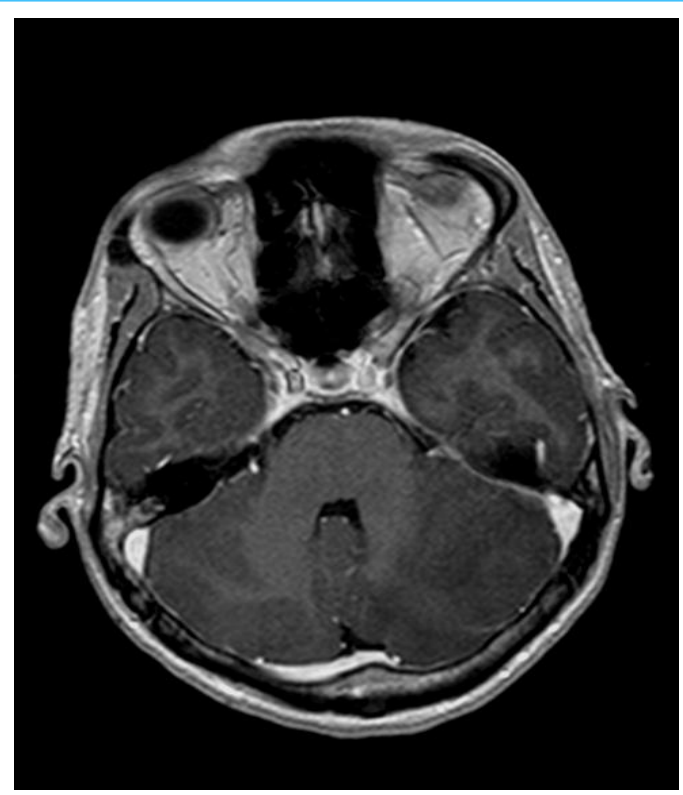

Figure 16: T1 weighted post contrast image: There is no appreciable enhancement.

Diagnosis: Dysplastic cerebellar gangliocytoma.

\section{Discussion:}

- Dysplastic cerebellar gangliocytoma is also known as Lhermitte-Duclos disease, after the persons who first described it in 1920.

- It is a rare cerebellar lesion. Its origin is controversial and may be hamartomatous, neoplastic or congenital malformation. Association with Cowden's syndrome (multiple hamartomas syndrome) favours the hamartomatous hypothesis.

- It is a benign condition per se and imaging helps in making a definitive diagnosis due to the typical striated folial pattern, hence obviating biopsy.

- Imaging also helps in selecting the patients who need surgery which includes patients in whom mass effect and hydrocephalus are present.

- Imaging also helps in preoperative planning by showing extent of tumor and finally imaging is useful in follow up of these patients.

\section{CASE-II}

A 60-year-old man presented with a subacute occipital headache. The pain is moderate but does not relieve with common analgesics and does not resolve spontaneously over 6 weeks. Past medical history is remarkable for bronchogenic carcinoma for which he was operated 2 years ago and is now in chemoradiotherapy. Metastatic survey 6 months before onset of headache showed no evidence of metastatic disease.

Neuroimaging is shown in following figures: 


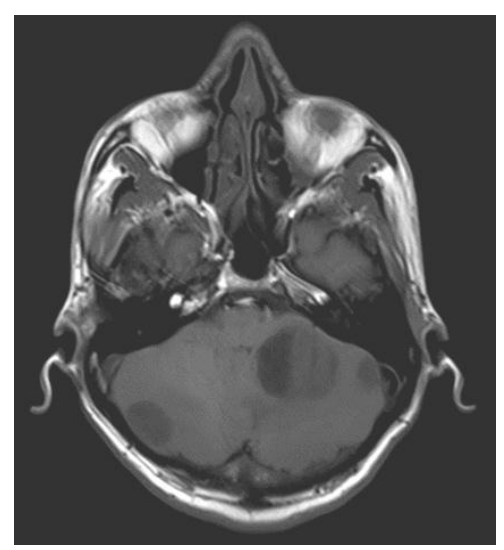

Figure 17: T1 weighted image: There are multiple well circumscribed round iso to hypointense lesions in both cerebellar hemispheres.

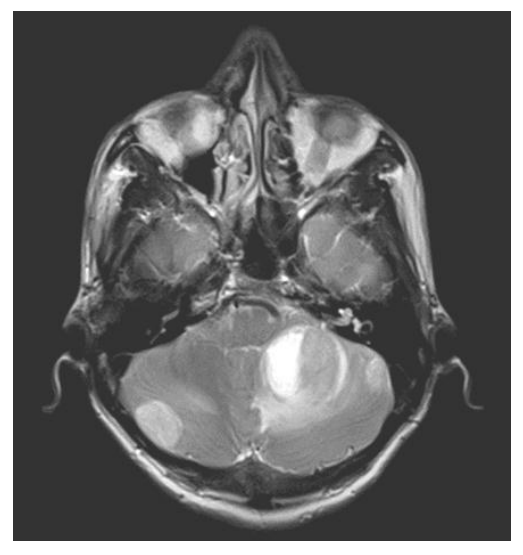

Figure 18: T2 weighted axial image: The lesions are slightly hyperintense to normal cerebellar tissue with mixed intensity in the larger lesion.

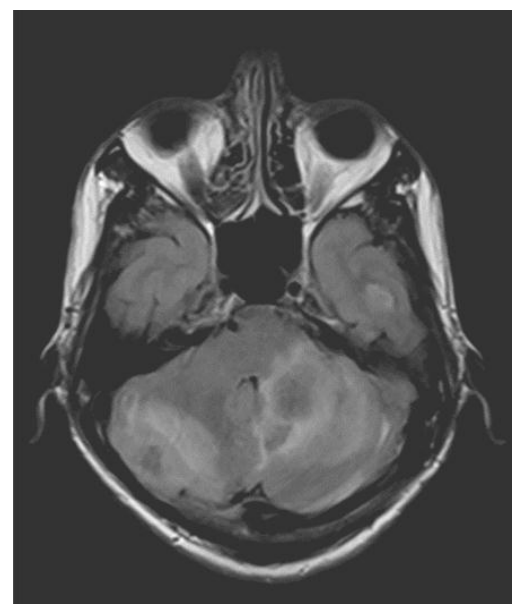

Figure 19: Fluid attenuated inversion recovery (FLAIR) axial image: The lesions are isointense to cerebellar tissue with moderate perilesional oedema. 


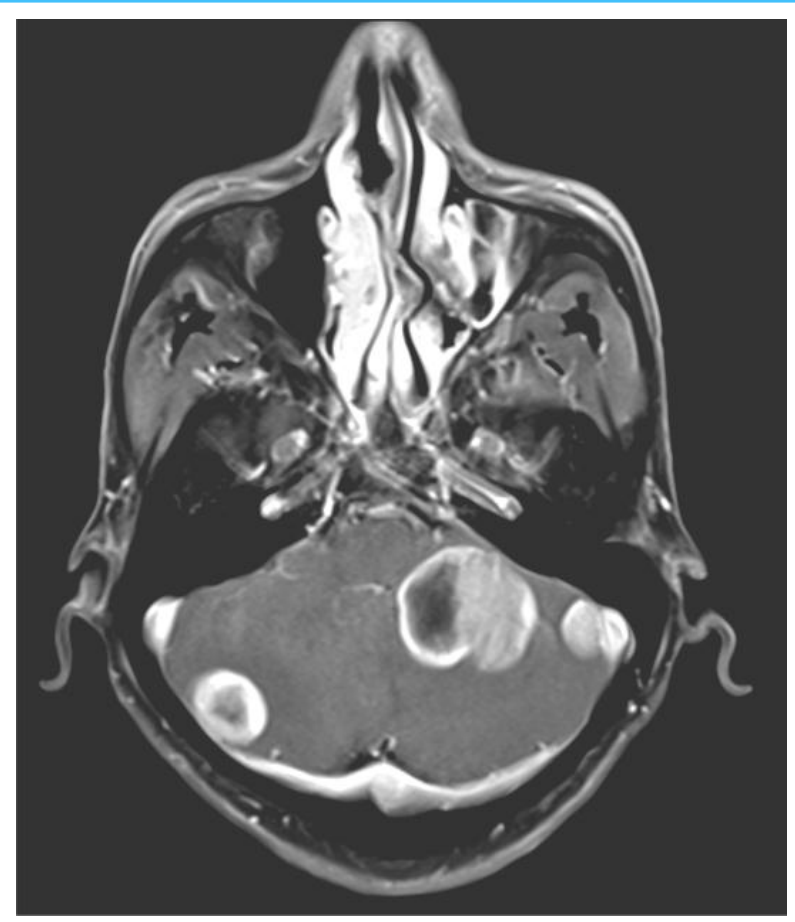

Figure 20: T1 weighted post contrast axial image: The lesions show intense nodular and ring enhancement.

Diagnosis: Metastasis

\section{Discussion:}

- A medical history of recent malignancy combined with round, enhancing lesions is highly suggestive of metastasis.

- The imaging characteristics of metastatic lesions include multiple nodular and ring enhancing lesions, commonly found at the gray-white matter interface.

- The most common infratentorial neoplasm in adults is a metastatic lesion. However, metastatic lesions are more commonly supratentorial.

- Though the presence of multiple lesions favors metastases versus a primary brain tumor, occasionally metastatic lesions will present as a solitary lesion.

- Metastatic lesions can present as calcified, hemorrhagic, or dural-based lesions. 


\section{CASE III}

A 45-year-old man presents with increasing sensory neural hearing loss and facial pain on the left side. Initial CT scan (not shown here) showed a cystic lesion isodense to CSF and a diagnosis of arachnoid cyst was made. The patient was followed with MRI due to persistent facial pain.

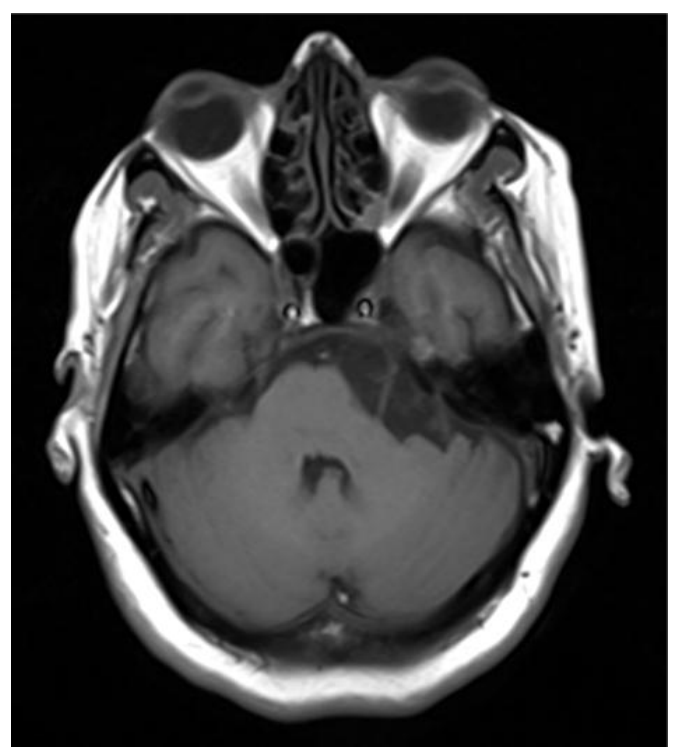

Figure 21: T1 weighted axial image: showing an extraaxial hypointense lesion in the left cerebellopontine angle.

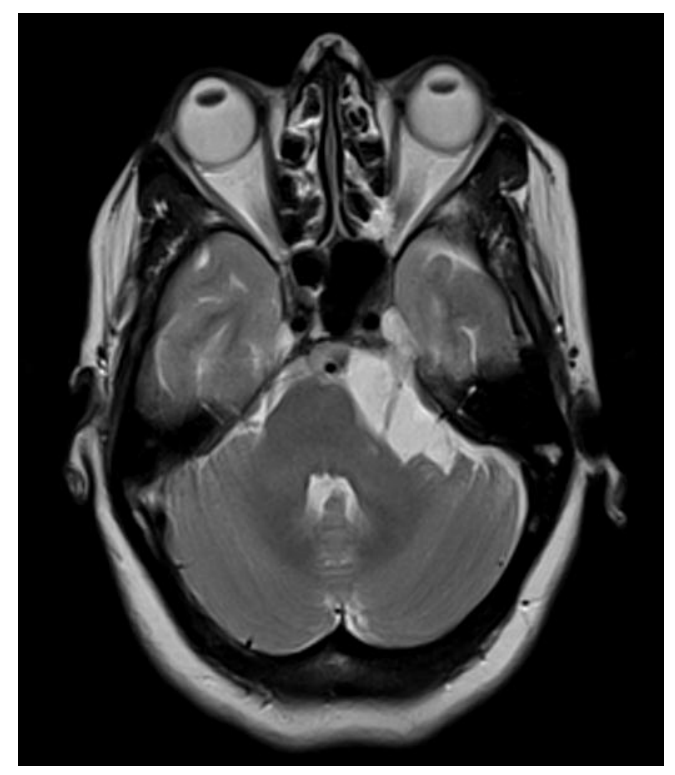

Figure 22: T2 weighted axial image: the lesion appears hyperintense. 


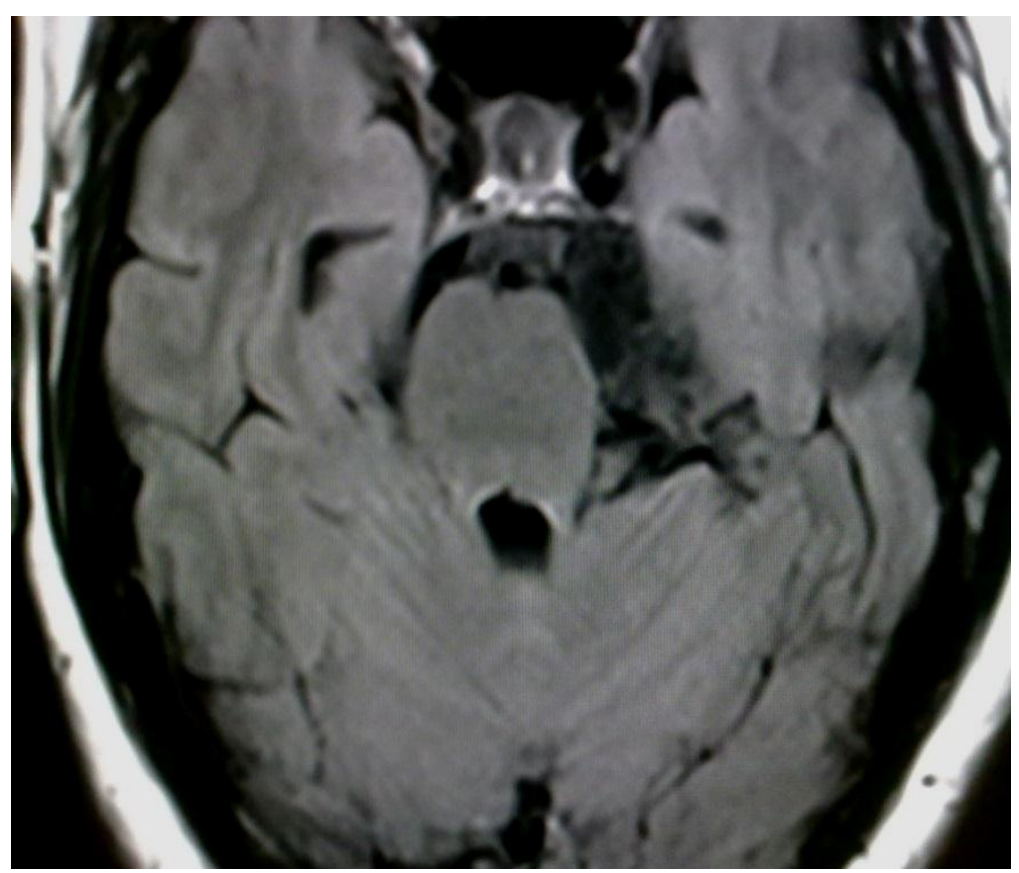

Figure 23: Fluid attenuated inversion recovery (FLAIR) image: The lesion displays heterogenous signal. Partly it is isointense to the cortex and partly it is hypointense to cortex but hyperintense to CSF.

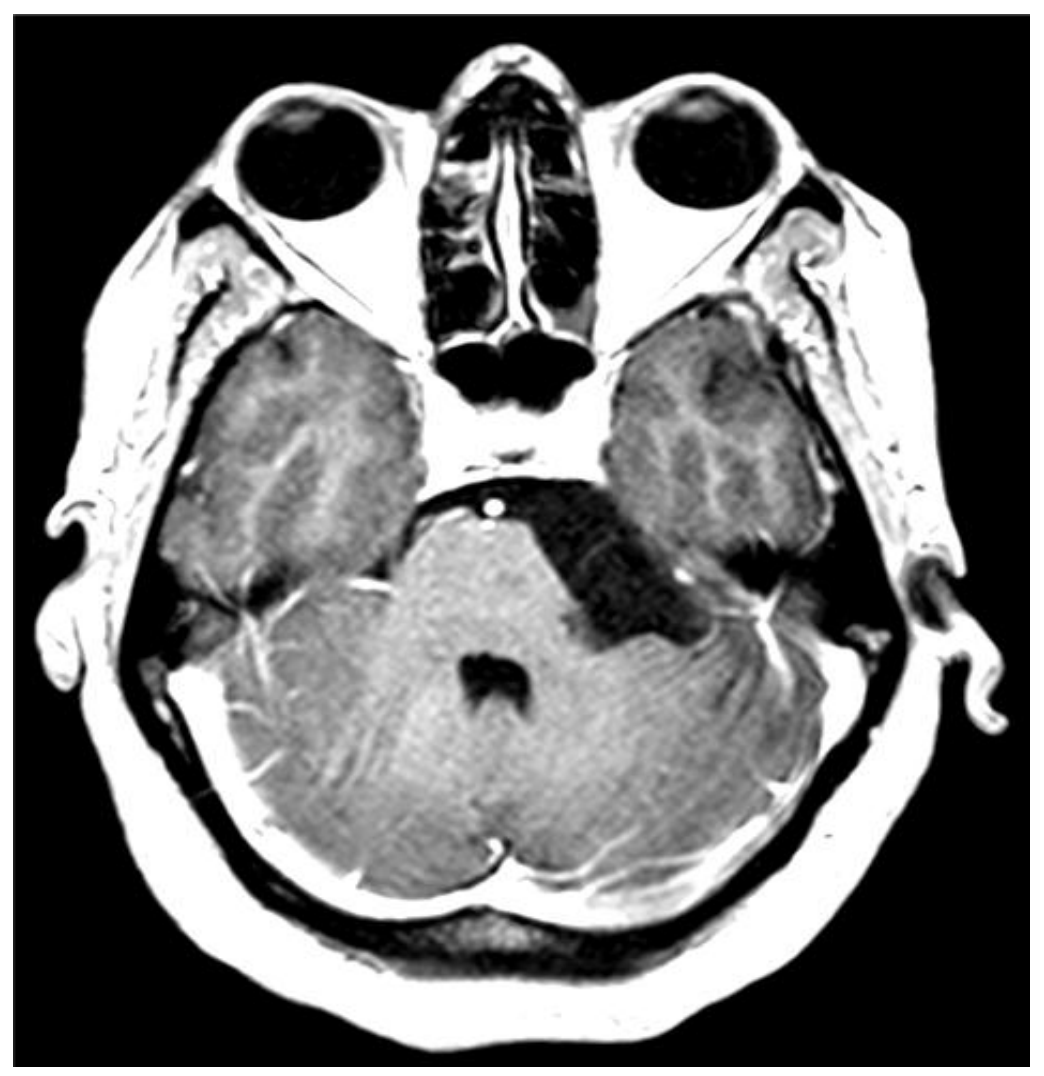

Figure 24: Postcontrast image: There is no appreciable enhancement. 
Diagnosis: Epidermoid

\section{Discussion:}

- The primary differential in this case of a cystic extra-axial mass lesion in the prepontine cistern is an arachnoid cyst or an epidermoid cyst.

- Epidermoid tumors, like arachnoid cysts are benign lesions.

- They tend to insinuate themselves between blood vessels and nerves.

- Epidermoid tumors usually will be hyperintense to the normal cerebrospinal fluid on T1 and T2 weighted images, as opposed to arachnoid cysts, which will follow the cerebrospinal fluid on all sequences.

- However epidermoid tumors can most easily be distinguished from arachnoid cysts on FLAIR images. Epidermoid cysts have high signal on FLAIR images.

\section{CASE IV:}

A 7-year-old child reports the subacute onset of headache, generalized weakness, and psychomotor slowing without focal neurological deficits.

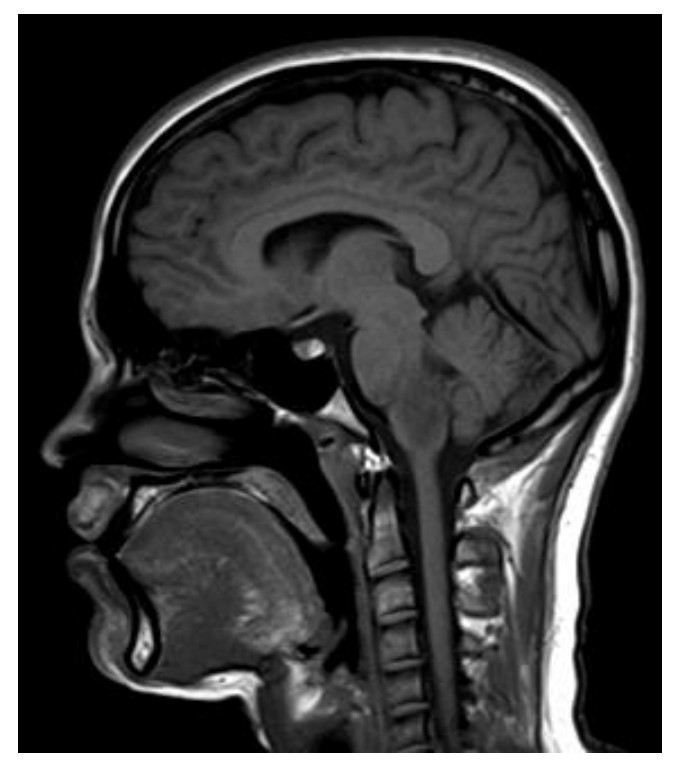

Figure 25: T1 weighted sagittal image: There is a diffuse hypointense lesion in the brainstem. 

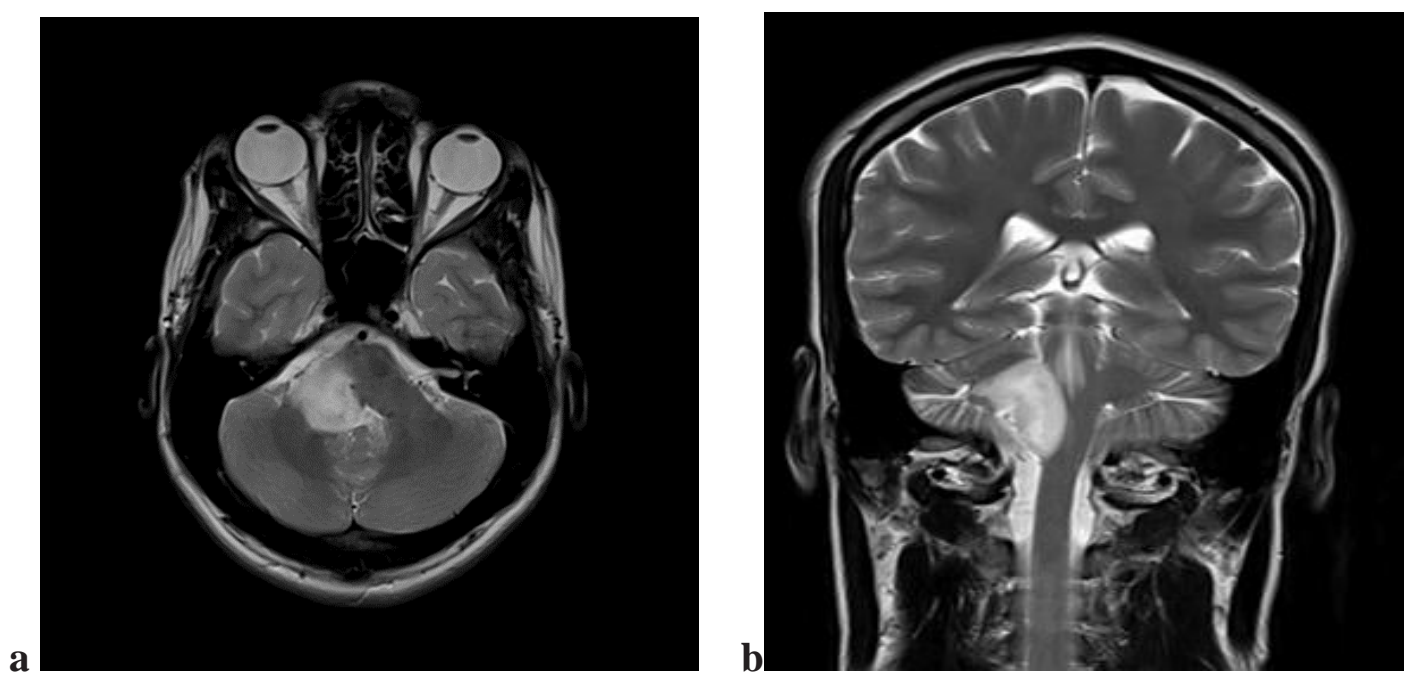

Figure 26a\&b: T2 weighted axial/coronal image: the lesion appears hyperintense and is located intraaxially within the brainstem predominantly on the right side.
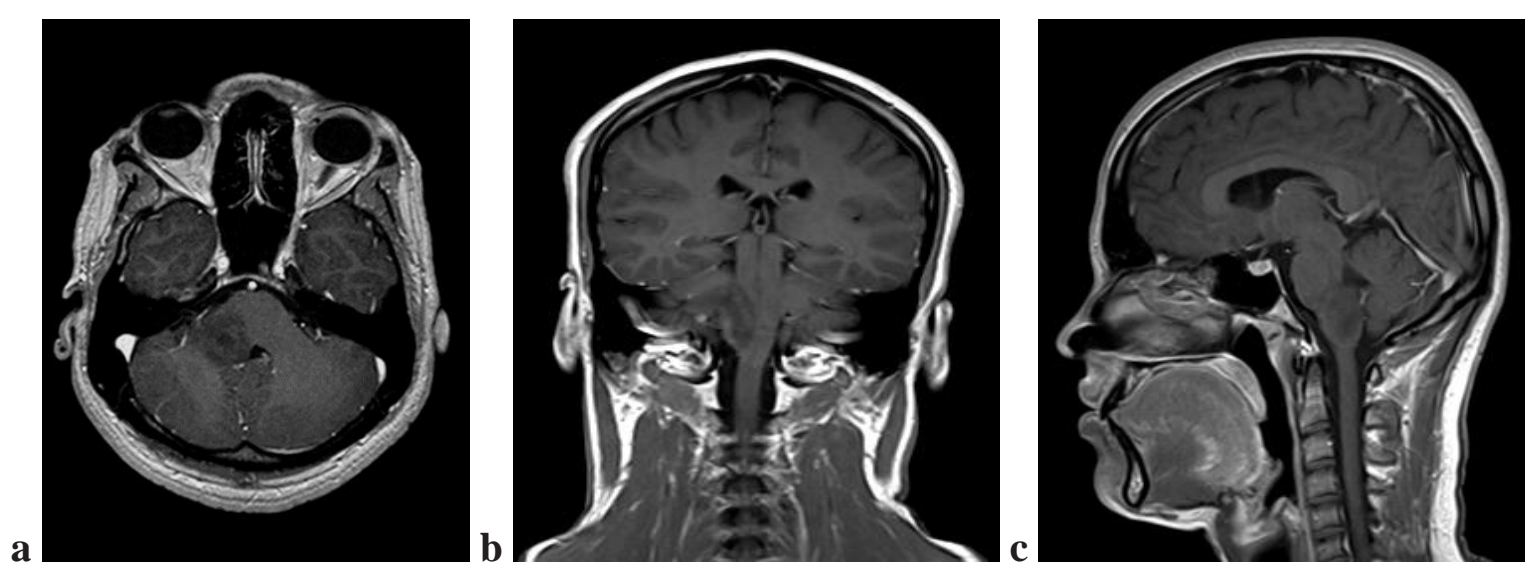

Figure 27a, b \& c: T1 weighted post contrast axial/coronal/sagittal images: There is no significant enhancement.

Diagnosis: Brainstem Glioma

\section{Discussion:}

- $\quad$ Brainstem gliomas constitute $10-20 \%$ of pediatric CNS tumors.

- Astrocytomas are the most common histologic type of brainstem tumor.

- Most brainstem gliomas are diffuse lesions that are low signal on T1 weighted images, high signal on T2 weighted images and have variable enhancement patterns.

- These tumors are usually malignant fibrillary astrocytomas (WHO grade III or IV).

- $\quad$ Some brain stem gliomas will have a focal well defined MRI appearance, and are usually lower grade (WHO grade I or II) tumors. 
- Exophytic tumors can either grow dorsally into the fourth ventricle (usually low grade) or ventrolaterally as in this case.

- Usually such ventrally directed exophytic tumors are of a high histologic grade.

CASE V:

A 50-year-old man presented with headaches and intermittent episodes of dizziness.

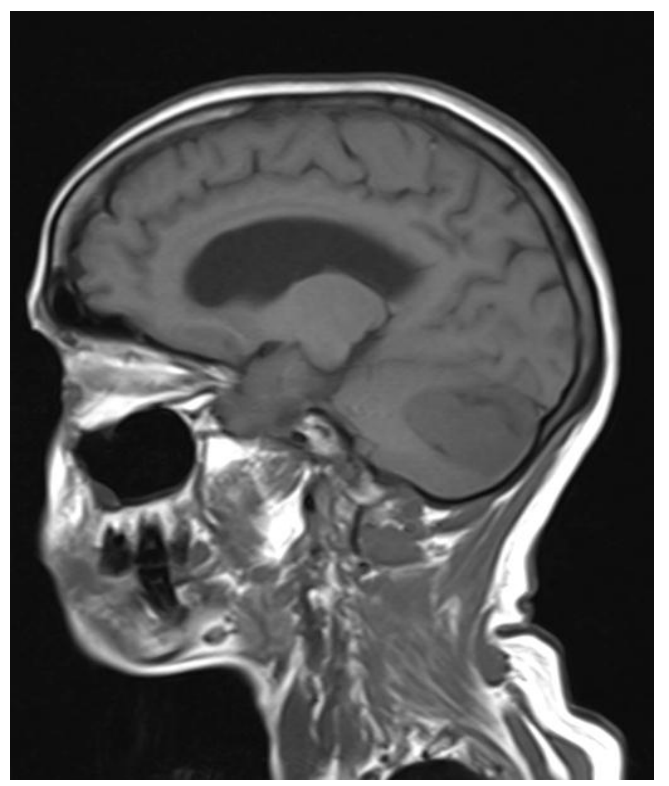

Figure 28: T1 weighted sagittal image: There is a well-defined lobulated hypointense lesion is seen in the cerebellum.
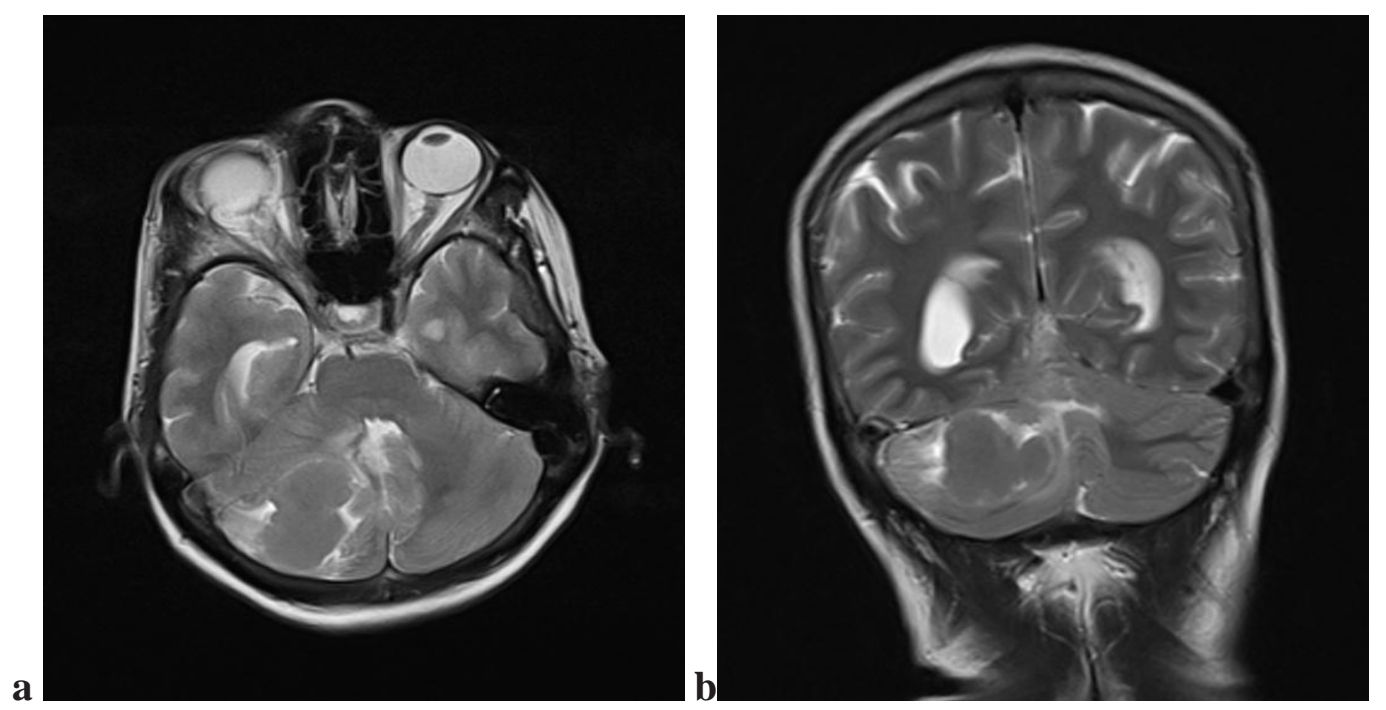

Figure 29a\&b: T2 weighted axial/coronal image: The lesion appears isointense to normal brain tissue and is surrounded by hyperintense oedema. 


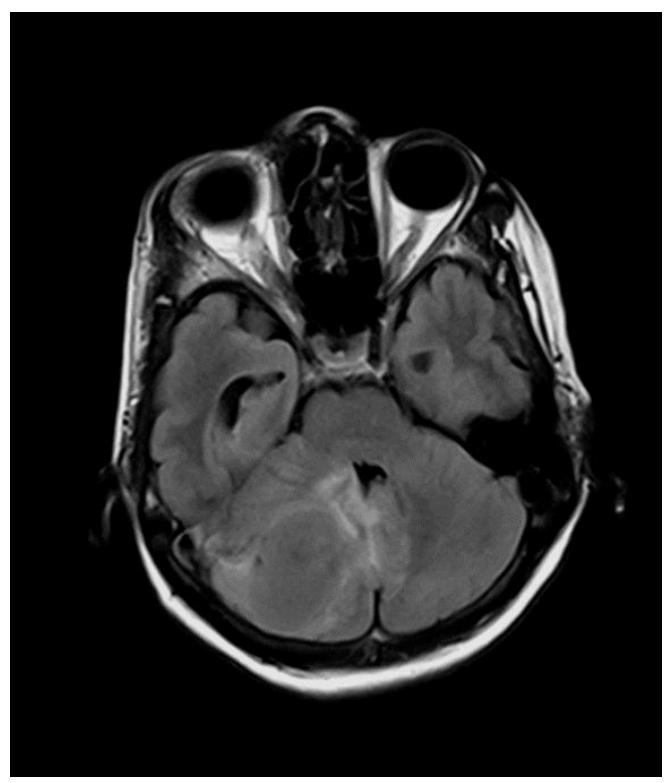

Figure 30: Fluid Attenuated Inversion Recovery (FLAIR) image: The lesion is seen more distinctly from the surrounding oedema.
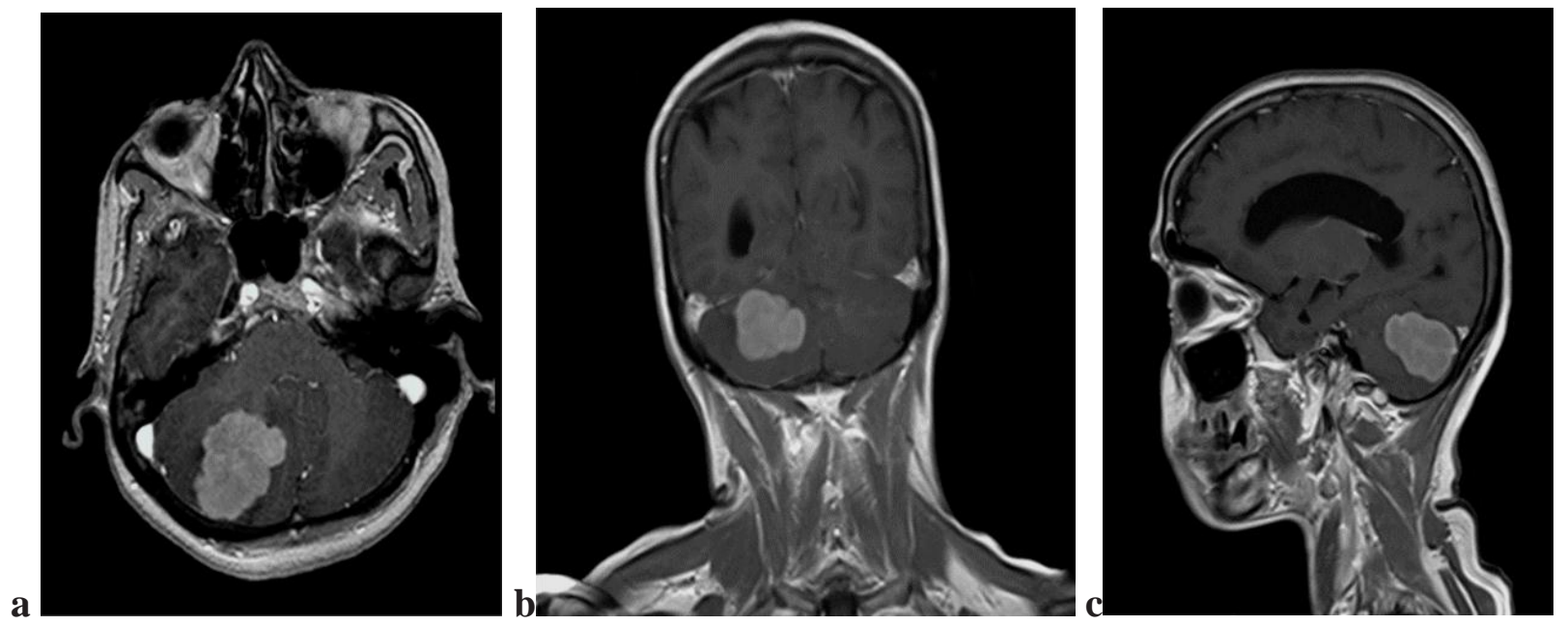

Figure 31a,b\&c: T1 weighted post contrast axial, coronal and sagittal images: the lesion shows homogenous intense enhancement.

Diagnosis: Atypical Hemangioblastoma

\section{Discussion:}

- Hemangioblastomas are benign neoplasms of endothelial origin that can occur sporadically or in association with Von Hippel Lindau disease (4-20\%).

- They are the most common infratentorial tumors of adult patients and are characterized by a hypervascular mural nodule that abuts the pia.

- In addition to the cerebellum these tumors can be found in the spine, brainstem and cerebrum. 
- On MRI these lesions most commonly are cystic lesions with a hypervascular mural nodule.

- They can be solid tumors with or without a central cystic region.

- Treatment is surgical resection, sometimes with preoperative embolization.

\section{CASE VI:}

61-year-old man presented with a 2-year history of progressive left-sided hearing loss.

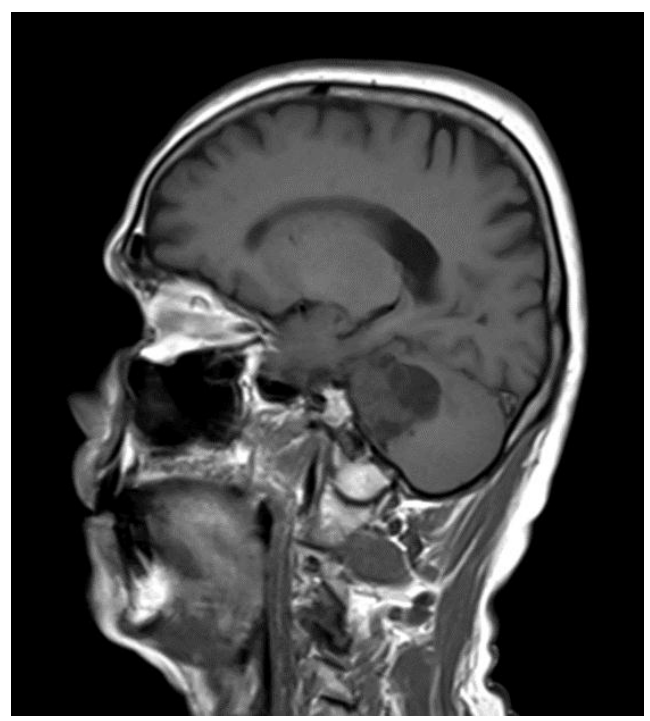

Figure 32: T1 weighted sagittal image: mixed intensity lesion in posterior fossa.
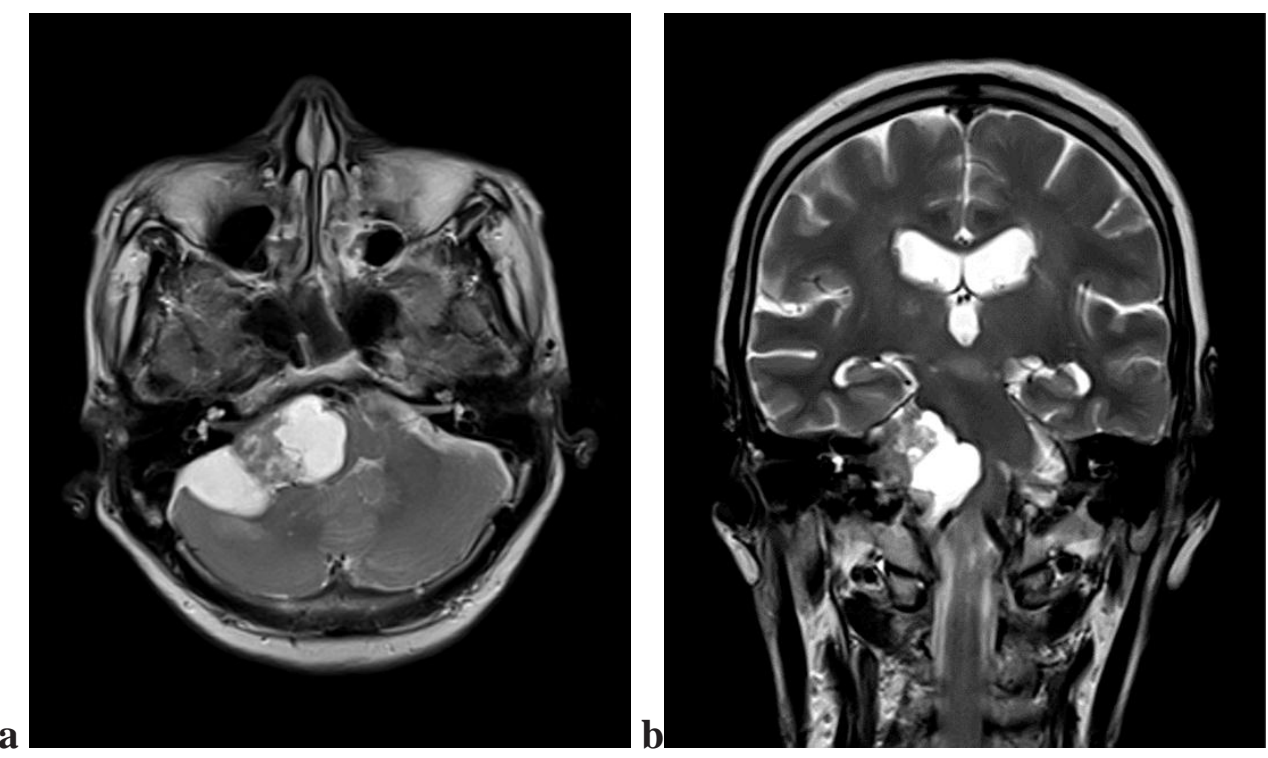

Figure 33a\&b: T2 weighted axial/coronal images: the lesion is heterogenous with solid and cystic areas. There is an extension into the internal auditory canal. 


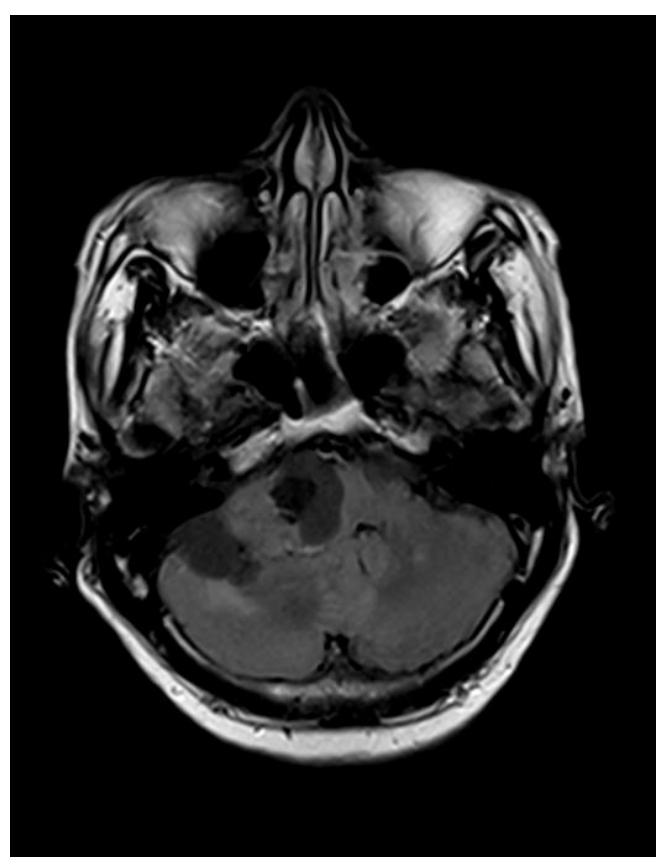

Figure 34: Fluid Attenuated Inversion Recovery (FLAIR) image: The lesion has mixed intensity with both solid and cystic areas.
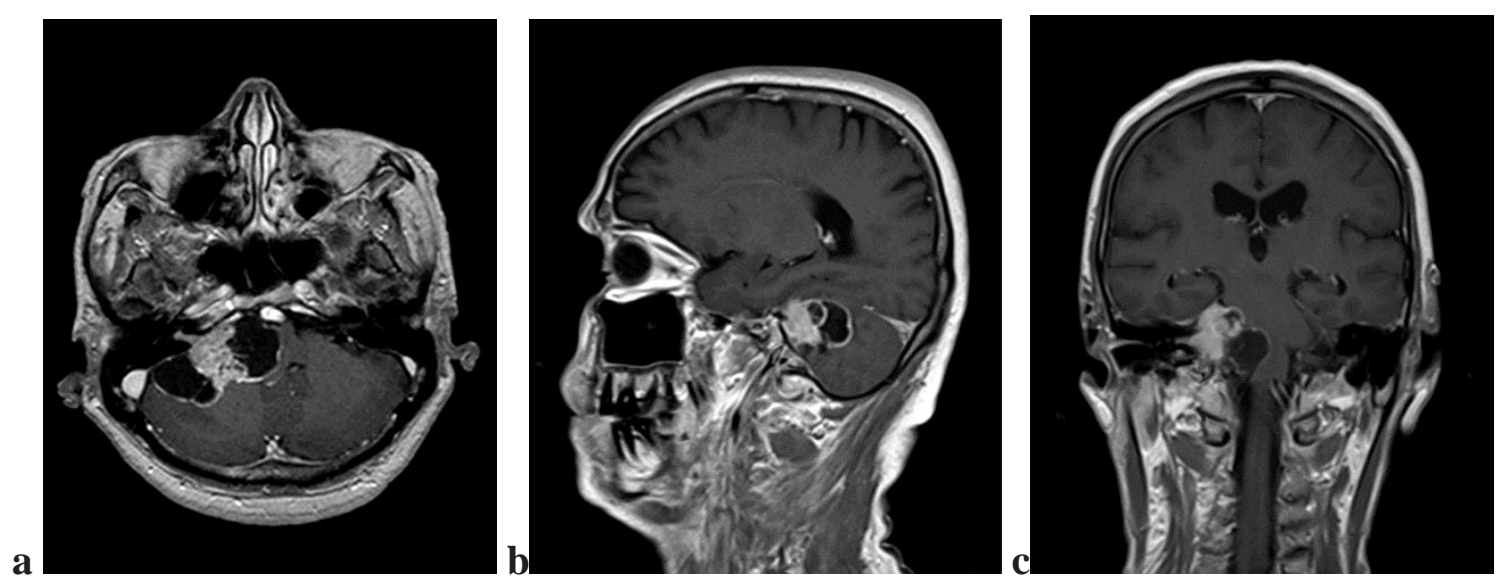

Figure 35 a, b \& c: T1 Weighted post contrast axial/sagittal/coronal images: There is intense enhancement of the solid component, walls of the lesion and the intracanalicular portion giving the typical "ice cream cone" appearance.

Diagnosis: Acoustic schwannoma.

\section{Discussion:}

- Vestibular schwannomas typically arise from Schwann cells lining the inferior vestibular nerve.

- These benign tumors are the most common mass lesions in the CPA, accounting for $70 \%$ to $80 \%$ of all masses in this region.

- The tumor most often presents in the fifth and sixth decades of life. 
- Symptomatic patients typically present with unilateral sensorineural hearing loss, tinnitus and/or disequilibrium.

- $\quad$ Bilateral VS are diagnostic of NF 2.

- While the classic ice-cream-cone-shaped VS involves the IAC (cone) with extension into the CPA cistern (ice-cream scoop), isolated intracanalicular and purely intracisternal lesions can also occur.

- Once the diagnosis of VS is established, imaging plays a key role in both treatment planning and follow up. Treatment strategies include watchful waiting, radiotherapy and surgery.

\section{CASE VII:}

A 25-year-old girl presented with headache, nausea and vomiting. Physical examination revealed gait ataxia and nystagmus. Fundus examination showed papilloedema. MRI was performed:

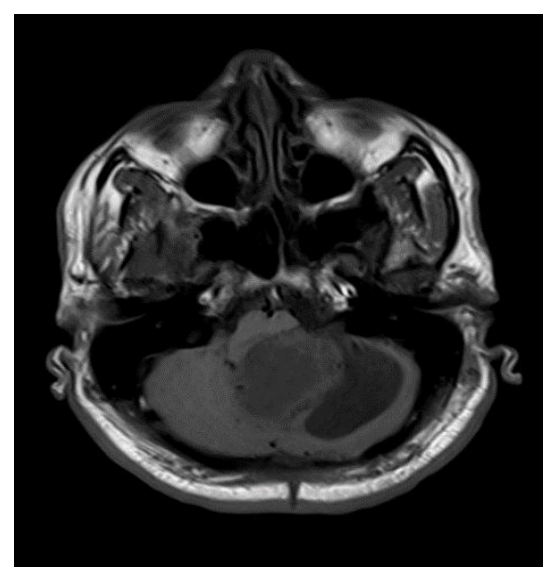

Figure 36: T1 weighted axial image: Mixed intensity lesion in the left cerebellar hemisphere and vermis. The lesion has solid and cystic/necrotic areas. Compressed fourth ventricle and brainstem.
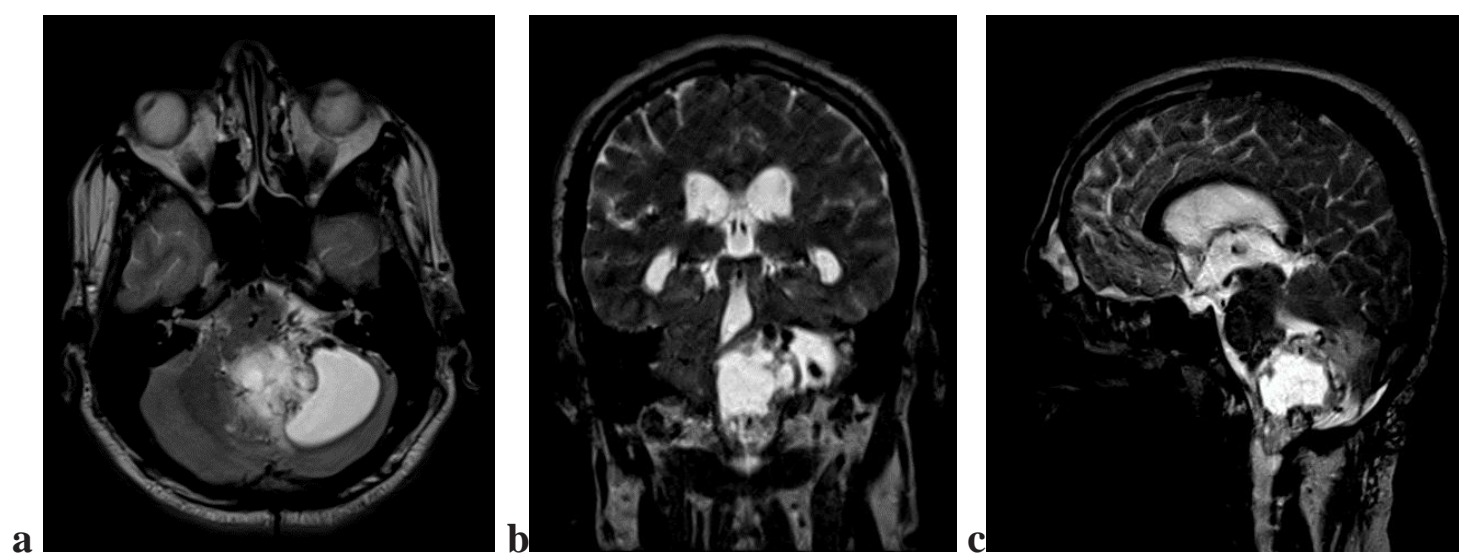

Figure 37a, b \& c: T2 weighted axial/coronal/sagittal images: Moderate oedema surrounding the heterogeneous lesion. Herniation of cerebellar tonsils and the tumor itself are through the foramen magnum. Compression of the fourth ventricle and resultant hydrocephalus. 


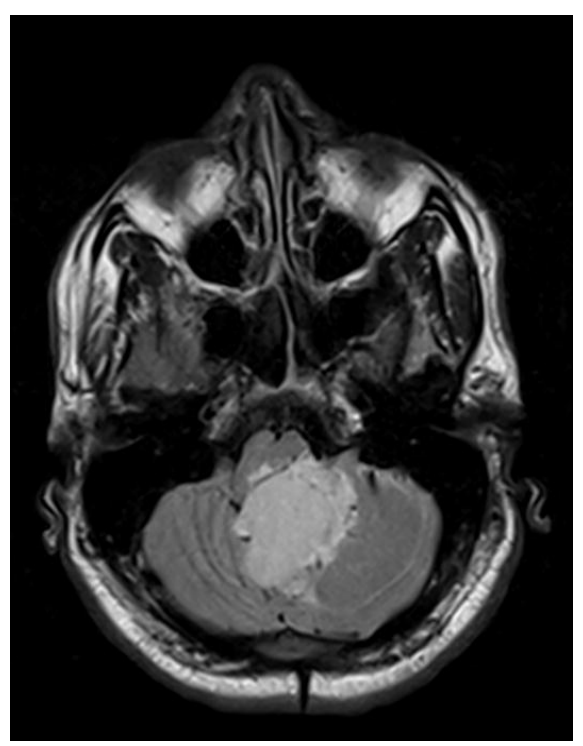

Figure 38: Fluid Attenuated Inversion Recovery (FLAIR) image: The solid component appears hyperintense and the cystic component appears isointense to the normal cerebellar tissue.
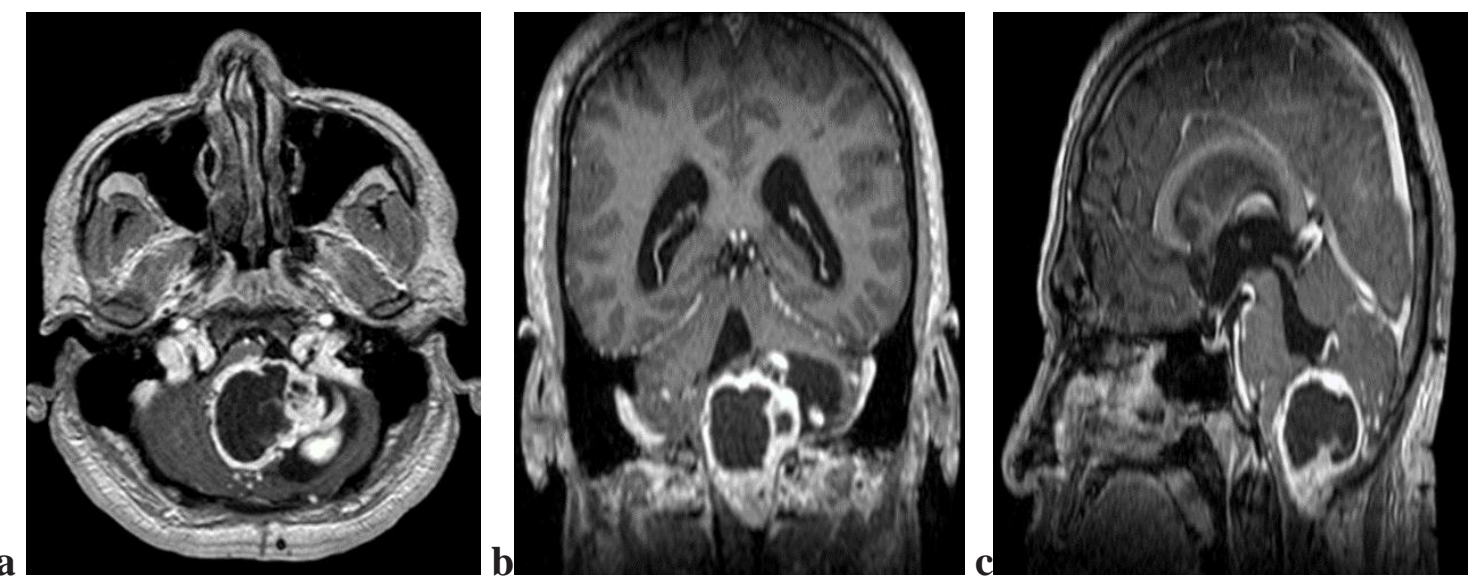

Figure 39 a, b \& c: T1 weighted post contrast axial/coronal/sagittal images: There is intense but heterogeneous enhancement.

Diagnosis: Anaplastic (grade III) astrocytoma.

\section{Discussion:}

- Anaplastic astrocytoma is a diffusely infiltrating astrocytoma with focal or dispersed anaplasia, and a marked proliferative potential.

- Anaplastic astrocytomas arise from low-grade astrocytomas, but are also diagnosed at first biopsy without indication of a less malignant precursor lesion.

- They have an intrinsic tendency for malignant progression to glioblastoma.

- Clinical presentation, patients may have a history of headache (tension-type 77\%, migraine-type $9 \%$, other types 14\%). Headaches can result from increased ICP (mass 
effect, blocked CSF flow), which may lead to the classic triad of headache, nausea and papilledema. Headache with other symptoms including nausea, vomiting and loss of consciousness is suggestive of a brain tumor (increased ICP).

- Malignant astrocytomas are usually hypointense on T1-weighted images and enhance heterogeneously following contrast infusion, enhancing tumor can be distinguished from the surrounding hypointense signal of edema on T1-weighted sequences.

- Astrocytomas regardless of their histologic grade generally show increased T2 signal intensity.

- Management of a high-grade astrocytoma may include stereotactic biopsy.

- Following biopsy and tissue diagnosis, there are the treatment options of surgical resection, radiation therapy and chemotherapy.

\section{CONCLUSION}

- Magnetic Resonance imaging was found to be a highly sensitive imaging procedure in detecting the tumor. Its high soft tissue resolution and multiplanar capabilities made it possible to know the exact site, plane and extent of the tumor.

- The MR imaging diagnosis was found to correlate with the histopathologic diagnosis in most instances. The positive predictive value of MRI was found to be $92 \%$.

- MR imaging was found to be highly sensitive in evaluating tumor related complications. Fourth ventricle was compressed in 25 patients with hydrocephalus in 20 patients. Brainstem was compressed or rotated in 11 patients. Cerebellar herniation was seen in 4 patients.

- MRI showed the full extent of the tumor and involvement of the surrounding tissues, thus helping in the management of tumors.

\section{RECOMMENDATIONS:}

The MR imaging diagnosis was found to correlate with the histopathologic diagnosis in most instances. MR imaging was found to be highly sensitive in evaluating tumor related complications. MRI showed the full extent of the tumor and involvement of the surrounding tissues, thus helping in the management of tumors. Hence it is recommended to perform MRI in cases of increased intracranial hypertension to make early and accurate diagnosis possible and hence improve the patient management.

\section{REFERENCES}

1. Andrea Poretti, Avner Meoded, Thierry A.G.M. Huisman. Neuroimaging of Pediatric Posterior Fossa Tumors. J. Magn. Reson. Imaging. 2012;35:32-47.

2. Bronstein K.S. Epidemiology and classification of brain tumours. Crit. Care Nurs Clin North Amer. 1995;7:79-89.

3. Rickert CH, Probst-cousin S, Gullotta F. Primary intracranial neoplasm of infancy and early childhood. Childs Nerv Syst, 1997;13, 507-13.

4. Friedman HS, Oakes WJ, Bigner SH et al: Medulloblastoma. Tumor biological and clinical perspectives. J Neurooncol 1991;11:1-15. 
5. Cohen M.E., and Dufner, P.K. Tumours of the brain and spinal cord including leukemic infiltrates. In SwaimanK Fed, Pediatric Neurology Principles and Practice. St. Louis Mosby. 1991;945-950.

6. Brown MT, Friedman HS, Oakes WJ et al: Chemotherapy for pilocytic astrocytomas. Cancer 1993;71:3165-3172.

7. Bronstein K.S. Epidemiology and classification of brain tumours. Crit. Care Nurs. Clin. North Amer. 1995;7:79-89.

8. Pollack IF. Pediatric brain tumours. Semin Surg Oncol, 1999;16:73-90.

9. Minimanoff RO, Dosoret Z DE, Lingood RM et al: Meningioma. Analysis of recurrence and progression following neurosurgical resection. J neurosur 1965;62:18-24.

10. Segall H D, Destian S, Nelson M D et al. (1990) CT and MR imaging in malignant gliomas. In: Apuzzo M L J (ed.) Malignant Cerebral Glioma. Park Ridge, IL: American Association of Neurological Surgeons; pp 63-78.

11. Osborn A G (1994) Diagnostic Radiology. St. Louis: Mosby. 234-235

12. Hustinx R \& Alavi A (1999) SPECT and PET imaging of brain tumors. Neuroimaging Clinics of North America 9:751-766

13. Lee BCP, Kneeland JB, Deck MDF, et al. Posterior fossa lesions: magnetic resonance imaging. Radiology 1984;153:137-143

14. Lee BCP, Kneeland JB, Deck MDF, et al. Posterior fossa lesions: magnetic resonance imaging. Radiology $1984 ; 153: 137-143$

15. Lee BCP, Kneeland JB, Walker RW et al. MR imaging of brainstem tumours. AJNR Am J Neuroradiology 1985; 6; 159-163.

16. George AE, Russel EJ, Kricheff II. White matter buckling: CT sign of extra axial intracranial mass. AJNR Am J Neuroradiol 1980;1:425-430

17. Barkovich AJ: Pediatric brain tumors, Sem US, CT, MR 1992;13:412-448

18. Lizak PF, Woodruff WW: Posterior fossa neoplasms: multiplanar imaging, Semin US, CT, MR 1992;13:182-206

19. Nemoto Y, Inoue Y, Fukuda T et al: Displacement of the quadrigeminal plate in tumors of the fourth ventricle: MR appearance, J Comp Asst Tomogr 1989;13:769-772

20. Lavaroni A, Leonard M: Neuroradiological diagnostics of brain tumors in adults, Riv di Neuroradiol 1993;(suppl 2):41-54

21. Bilaniuk LT. Adult infratentorial tumors. Semin Roentgenol. 1990 Apr; 25(2):155-73.

22. Raphael Guzman, Alain Barth, Karl-Olof Lövblad. Use of diffusion-weighted magnetic resonance imaging in differentiating purulent brain processes from cystic brain tumors. $\mathbf{J}$ Neurosurg 2002;97:1101-1107.

23. Kinuko Kono, Yuichi Inoue, Keiko Nakayama, Miyuki Shakudo, Michiharu Morino, Kenji Ohata, Kenichi Wakasa, Ryusaku Yamada. The Role of Diffusion-weighted Imaging in Patients with Brain Tumors. AJNR Am J Neuroradiol. June/July 2001;22:1081-1088

24. Kim M. Cecil, Blaise V. Jones. Magnetic Resonance Spectroscopy of the Pediatric Brain. Topics in Magnetic Resonance Imaging. 2001;12(6):435-452

25. Rehman A.U., Lodhi S., Murad S. Morphological Pattern of Posterior Cranial, Fossa Tumors. ANNALS. APR. - JUN. 2009;15(2):57-59

26. P.K. Wanyoike. Posterior cranial fossa tumours in children at kenyatta national hospital, nairobi. East African Medical Journal. May 2004;81(5):258-260 
27. Chang T, Teng MM, Lirng JF Posterior cranial fossa tumours in childhood. Neuroradiology. 1993;35(4):274-8.

28. Sarrazin J, Haclie O, Cordoliani Y. Cerebellopontine angle tumors in adults. J Radiol. 2000 Jun;81(6 Suppl):675-90.

29. Curnes JT. MR imaging of peripheral intracranial neoplasms: extraaxial vs intraaxial masses. J Comput Assist Tomogr.1987 Nov-Dec;11(6):932-7.

30. Mulkens TH, Parizel PM, De Schepper AM, van de Heyning PH, Forton GE, Martin JJ, Delaporte C. MRI of acoustic schwannoma: a retrospective study of 89 tumours.Rofo.1993Apr;158(4):362-7.

31. Izycka-Swieszewska E, Szurowska E, Kloc W, Rzepko R, Dubaniewicz-Wybieralska M, Skorek A, DrozyÅ,ska E, Stempniewicz M. Cerebellopontine angle tumours:radiologicpathologic correlation and diagnostic difficulties. Folia Neuropathol. 2006;44(4):274-81.

32. M Wakoh, H Yonezu, T Otonari, T Sano, K Matsuzaka, T Inoue and N Wada. Two cases of schwannoma with marked cystic changes. Dentomaxillofacial Radiology. 2005;34:4450

33. Maiuri F, Iaconetta G de Divitiis O, et al. Intracranial Meningioma: correlations between MR imaging and histology. Eur J Radiol. July 1999; 31(1):69-75.

34. Scott W. Atlas. Magnetic resonance imaging of the Brain and Spine, $3^{\text {rd }}$ ed. Baltimore: Williams \& Wilkins, 2002:701.

35. Takeguchi T, Miki H, Shimizu T, Kikuchi K, Mochizuki T, Ohue S, Ohnishi T. The dural tail of intracranial meningiomas on fluid-attenuated inversion-recovery images. Neuroradiology. 2004 Feb; 46(2):130-5.

36. Carlos Majos, Gracia Cucurella, Carlos Aguilera et al Intraventricular Meningioma and MR spectroscopic Findings in two cases. AJNR Am J Neuroradiol 1999;882-885.

37. Bonneville F, Savatovsky J, Chiras J.Imaging of cerebellopontine angle lesions: an update. Part 1: enhancing extra-axial lesions. EurRadiol. 2007 Oct;17(10):2472-82.

38. Dutt SN, Mirza S, Chavda SV, Irving RM. Radiologic differentiation of intracranial epidermoids from arachnoid cysts. Otol Neurotol. 2002 Jan;23(1):84-92.

39. Wasenko JJ, Rosenbloom SA, Estes M, Lanzieri CF, Duchesneau PM. Magnetic resonance of intracranial epidermoids. Eur J Radiol. 1991 Sep-Oct;13(2):103-6.

40. Kallmes DF, Provenzale JM, Cloft HJ, McClendon RE. Typical and atypical MR imaging features of intracranial epidermoid tumors. AJR Am J Roentgenol. 1997 Sep;169(3):8837.

41. Rodallec M, Colombat M, Krainik A, Kalamarides M, Redondo A, Feydy A. Diffusionweighted MR imaging and pathologic findings in adult cerebellar medulloblastoma. J Neuroradiology. 2004 Jun;31(3):234-7.

42. Qiu SJ, Zhang XL. MRI features of 13 cases of intracranial ependymoma. Di Yi Jun Yi Da Xue Xue Bao. 2003 Nov;23(11):1224-5.

43. Warmuth - Metz M, Kuhl J. Neuroradiological differential diagnosis in medulloblastomas and ependymomas: results of the HIT'91-study. Klin Padiatr. 2002 Jul-Aug;214(4):162-6.

44. Lee YY, Van Tassel P, Bruner JM, Moser RP, Share JC. Juvenile pilocytic astrocytomas: CT and MR characteristics. AJR Am J Roentgenol. 1989 June;152(6):1263-70.

45. Coakley KJ, Huston J 3rd, Scheithauer BW, Forbes G, Kelly PJ. Pilocytic astrocytomas: well-demarcated magnetic resonance appearance despite frequent infiltration histologically. Mayo Clin Proc. 1995 Aug;70(8):747-51. 
46. Koeller KK, Rushing EJ. From the archives of the AFIP: pilocytic astrocytoma: radiologicpathologic correlation. Radiographics. 2004 Nov-Dec;24(6):1693-708.

47. Zimmerman RA : Neuroimaging of primary brain stem gliomas. Diagnosis and course.Pediatr Neurosurg 1996;25:45-53

48. Lee SR, Sanches J, Mark AS, Dillon WP, Norman D, Newton TH. Posterior fossa hemangioblastoma: MR imaging. Radiology. 1989 May; 171(2):463-8.

49. Zhou CW, Zhang HM, Ouyang H. Intracranial metastasis of malignant tumors: clinical characteristics and MR imaging features. Zhonghua Zhong Liu Za Zhi. 2004 Sep;26(9):554-7.

50. Tsuruda J.S., Chew W.M., Moseley M.E., Norman D.: Diffusion Weighted MR imaging of the brain: value of differentiating between extraaxial cysts and epidermoid tumors. AJNR Am J Neuroradiol. 1990;11:925-931.

51. Chen S, Ikawa F, Kurisu K, Arita K, Takaba J, Kanou Y. Quantitative MR evaluation of intracranial epidermoid tumors by fast fluid-attenuated inversion recovery imaging and echo-planar diffusion-weighted imaging. AJNR 2001;22:1089-1096.

52. Tadeusz W. Stadnik, Cristo Chaskis, Alex Michotte, Wael M. Shabana, Katrijn van Rompaey, Robert Luypaert, Lubos Budinsky, Vladimir Jellus, and Michel Osteaux. Diffusion-weighted MR Imaging of Intracerebral Masses: Comparison with Conventional MR Imaging and Histologic Findings. AJNR Am J Neuroradiol 2001 May;22:969-976,

53. Rumboldt Z, Camacho DL, Lake D, Welsh CT, Castillo M. Apparent diffusion coefficients for differentiation of cerebellar tumors in children. AJNR Am $J$ Neuroradiol 2006;27:1362-1369.

54. Schneider JF, Confort-Gouny S, Viola A, et al. Multiparametric differentiation of posterior fossa tumors in children using diffusion- weighted imaging and short echo-time 1H-MR spectroscopy. J Magn Reson Imaging 2007;26:1390-1398.

55. Bulakbasi N, Guvenc I, Onguru O, et al. The added value of the apparent diffusion coefficient calculation to magnetic resonance imaging in the differentiation and grading of malignant brain tumors. J Comput Assist Tomogr 2004; 28:735-46.

56. Yamasaki F, Kurusi K, Satoh K, et al. Apparent diffusion coefficient of human brain tumors at MR imaging. Radiology 2005; 235:985-91

57. Murakami et al Grading astrocytic tumors by using apparent diffusion coeeficient parameters Radiology 2009;251(3):838-845.

58. Klatzo I. Presidental address: neuropathological aspects of brain edema. J Neuropathol Exp Neurol 1967;26:1-4.

59. Tien R, Felsberg G, Friedman H, Brown M, MacFall J. MR imaging of high-grade cerebral gliomas: value of diffusionweighted echoplanar pulse sequences. AJR Am J Roentgenol 1994;162:671-677.

60. Brunberg J, Chenevert T, McKeever P, et al. In vivo MR determination of water diffusion coefficients and diffusion anisotropy: correlation with structural alteration in gliomas of the cerebral hemispheres. AJNR Am J Neuroradiol 1995;16:361-371.

61. Plate KH, Breier G, Weich HA, Risau W. Vascular endothelial growth factor is a potential tumour angiogenesis factor in human gliomas in vivo. Nature 1992;359:845-848.

62. Folkman J. Seminars in medicine of the Beth Israel Hospital, Boston: clinical applications of research on angiogenesis. N Engl J Med 1995; 333:1757-1763. 
63. Leon PS, Folkerth RD, Black PM. Microvessel density is a prognostic indicator for patients with astroglial brain tumors. Cancer 1996; 77:362-372.

64. Glantz MJ, Burger PC, Herdon JE II, et al. Influence of type of surgery on the histologic diagnosis in patients with anaplastic gliomas. Neurology 1991;41:1741-1744.

65. Belliveau JW, Rosen BR, Kantor HL, et al. Functional cerebral imaging by susceptibility contrast NMR. Magn Reson Med 1990;14:538-546.

66. Rosen BR, Belliveau JW, Aronen HJ et al. Susceptibility contrast MR imaging of cerebral blood volume: human experience. Magn Reson Med 1991;22:293-299.

67. Rosen BR, Belliveau JE, Vevea JM, Brady TJ. Perfusion imaging with NMR contrast agents. Magn Reson Med 1990;14:249-265.

68. Aronen HJ, Gazit EI, Louis DN, et al. Cerebral blood volume maps of gliomas: comparison with tumor grade and histologic findings. Radiology 1994;191:41-51.

69. Brant-Zawadski M. Pitfalls of contrast enhanced imaging in the nervous system. Magn Reson Med 1991;22:243-248.

70. Panigrahy, M.D. Krieger, I. Gonzalez-Gomez, X. Liu, J.G. McComb, J.L. Finlay, M.D. Nelson, Jr. F.H. GillesS. Blu"ml. Quantitative Short Echo Time 1H-MR Spectroscopy of Untreated Pediatric Brain Tumors: Preoperative Diagnosis and Characterization. AJNR Am J Neuroradiol Mar 2006;27:560-72

71. Meng Law, Soonmee Cha, Edmond A. Knopp, Glyn Johnson, John Arnett, Andrew W. Litt. High-Grade Gliomas and Solitary Metastases: Differentiation by Using Perfusion and Proton Spectroscopic MR Imaging. Radiology March 2002;222:715-721

72. Sutton LN, Wang Z, Gusnard D, et al. Proton magnetic resonance spectroscopy of pediatric brain tumors. Neurosurgery1992;31:195-202.

73. Kugel H, Heindel W, Ernestus RI, et al. Human brain tumors: Spectral patterns detected with localized H-1 MR spectroscopy. Radiology 1992;183:701-9.

74. Kee-Hyun Chang, In Chan Song, Sung Hyun Kim, Moon Hee Han, Hong Dae Kim, Su Ok Seong, Hee Won Jung, and Man Chung Han. In Vivo Single-Voxel Proton MR Spectroscopy in Intracranial Cystic Masses. AJNR Am J Neuroradiol March 1998;19:401405,

75. Kim M. Cecil, Ph.D. and Blaise V. Jones, M.D. Magnetic Resonance Spectroscopy of the Pediatric Brain. Topics in Magnetic Resonance Imaging 2001;12(6): 435-452

76. Janine M. Lupo, Suchandrima Banerjee, Kathryn E. Hammond, Douglas A.C. Kelley, Duan X, Susan M. Chang, Daniel B. Vigneron, Sharmila Majumdar, Sarah J. Nelson. GRAPPA-based susceptibility-weighted imaging of normal volunteers and patients with brain tumor at 7 T. Magnetic Resonance Imaging 2009;27: 480-488 